\title{
A frequency approach to identifying asteroid families
}

\author{
V. Carruba ${ }^{1}$ and T. A. Michtchenko ${ }^{2}$
}

1 IPD, UNIVAP, São José dos Campos, SP, 12244-000, Brazil

e-mail: valerio@univap.br

2 IAG, USP, São Paulo, SP, 05508-900, Brazil

e-mail: tatiana@astro.iag.usp.br

Received 20 April 2007/ Accepted 17 August 2007

\section{ABSTRACT}

\begin{abstract}
Context. Secular resonances usually have a complicated three-dimensional structure in $a-e-i$ (or $a-e-\sin (i))$ space, which is not easily represented in a two-dimensional projection. As a consequence, the classic approach to identifying asteroid families fails in some cases to identify objects that have migrated in such resonances because of the Yarkovsky effect.

Aims. We propose an alternative approach by identifying asteroid families in the proper frequency domain $(n, g, g+s)$ rather than in the proper element domain $(a, e, \sin (i))$.

Methods. We applied the HCM method in the proper frequency domain $(n, g, g+s)$ to identify four of the largest asteroid families: Vesta, Eunomia, Eos, and Koronis. We compared our results with those obtained with the classical method. In addition, we applied the extended metrics in the domain of asteroid colors in both proper element and frequency domains.

Results. The frequency approach to determining families is an excellent tool for (i) more easily identifying the effect of nonlinear secular resonances on families and (ii) connecting to the family of origin objects that have migrated via the interplay of the Yarkovsky effect and nonlinear secular resonances.
\end{abstract}

Key words. minor planets, asteroids - celestial mechanics

\section{Introduction}

Asteroid families are groups of minor planets sharing a common collisional origin. Since the work of Hirayama (1918), it has been noticed that clusters of objects appear when the proper orbital elements of asteroids (proper semi-major axis $a$, eccentricity $e$, and inclination $\left.i^{1}\right)$ are plotted in the $a-e$ or $a-\sin (i)$ planes. Zappalà et al. (1990) introduced the hierarchical clustering method (HCM hereafter) to identify neighbors in proper element space and separate asteroid families from objects in the background. This method has been successively used by several authors (Zappalà et al. 1993; Bendjoya \& Zappalà 2002; Nesvorný et al. 2005, just to quote some of the works on the subject), and it is currently one of the most used tools for determining asteroid families ${ }^{2}$.

\footnotetext{
${ }^{1}$ Unlike osculating orbital elements, which respond to short-period perturbations, proper elements determined via analytical (Milani \& Knežević 1994) or numerical (Knežević, 2002) methods are nearly constant over long-time spans.

${ }^{2}$ We must make a distinction between the original family and the family determined with the classical HCM and other methods described in this article. When the asteroid family was formed, their members were thrown in a region already populated by other objects, sometimes having different superficial compositions. Also, the orbits of some of the family members evolved due to the several gravitational and nongravitational effects described in the article. Therefore, when applying methods such as the HCM, we find clusters of objects in the current proper element or frequency space that may or may not have the same origin. Also, these methods may miss some of the asteroids that evolved farther away from the family core. When using the word family we will usually refer to the agglomeration of objects found with the methods described in this article, keeping in mind that the relationship of these
}

However, many long-lasting dynamical effects can modify the orbits of members of asteroid families after the collisional event that originated them. Contrary to what was believed in the early 90s, asteroid proper elements can be significantly modified due to collisional evolution (O'Brien \& Greenberg 2005), thermal effects such as the Yarkovsky and YORP effects (Vokrouhlický 1999; Vokrouhlický \& Čapek 2002), repeated close encounters with massive asteroids (Carruba et al. 2003, 2007a), capture into mean-motion and secular resonances (Gladman et al. 1997), and the interplay of any of these mechanisms.

In particular, nonlinear secular resonances have recently assumed a significant role in asteroid dynamics. Secular resonances are commensurabilities among the frequencies of variation in the longitudes of perihelion, or in the longitudes of nodes or in both of an asteroid and the planets. Several groups (Bottke et al. 2001, for the Koronis family; Vokrouhlický et al. 2002, 2006, for the Eos family; Carruba et al. 2005, 2007a,b, for several V-type asteroids outside the Vesta and Eunomia families, among others) have demonstrated that the interplay of the Yarkovsky effect and nonlinear secular resonances has sculpted the shape of observed families and it is responsible for the diffusion of family members that today are no longer recognizable as such when methods like the classical HCM are used.

Nonlinear secular resonances usually have complicated three-dimensional structures in the proper $a-e-\sin (i)$ space, and are not easily represented in a two-dimensional projection (see Sect. 2). As a consequence, the classical Zappalà approach in some cases fails to identify objects that have migrated in such

clusters with the original agglomeration of objects created in the collisional event is generally a very complicated one. 


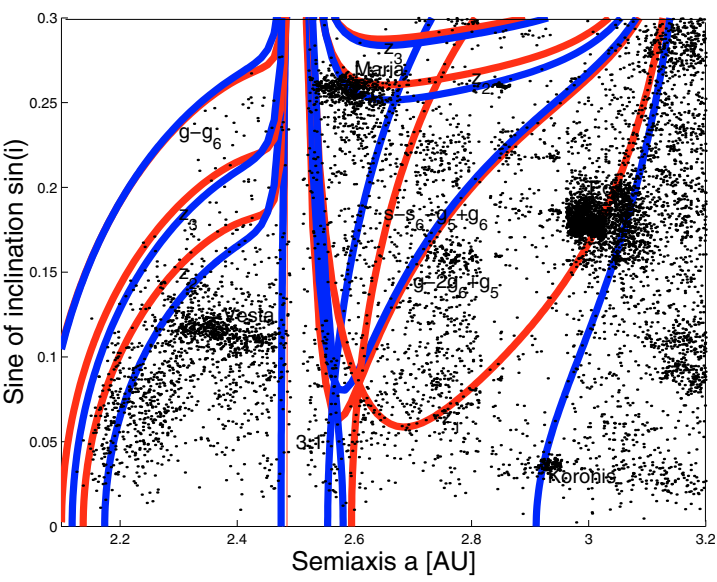

Fig. 1. The location of the main secular resonances in the asteroid belt. The secular resonances obtained for the fixed value 0.10 of the proper eccentricity are superimposed to the proper elements $a$ and $\sin (i)$ of the asteroids with proper $e$ within the range of \pm 0.025 of the central value. The names indicate the main asteroid families in the region. The red lines display the locations of the resonances at the lower edge, while the blue curves show the same at the upper edge. See Figs. 7, 8 and 9 of Milani \& Knežević (1994) for more details.

resonances because of the Yarkovsky effect as family members, and, more generally, makes understanding the dynamics involving secular resonances more difficult than it should be.

We believe this problem may be overcome if we analyze asteroid families in the space of proper asteroid frequencies $(n, g, s)$, where $n$ is the asteroid mean-motion and $g, s$ are the asteroid secular frequencies of the pericenter and node, respectively ${ }^{3}$. Indeed, the complicated three-dimensional structure of secular resonances may be more easily represented in the proper $(g, g+s)$ plane, where secular resonances appear as vertical (resonances of pericenter), horizontal (resonances that contain the $(g+s)$ combination), and inclined lines (resonances of node and other linear combinations of pericenter and node), forming the so-called "Arnold web" of resonances. In this paper we show that, by applying the HCM procedure in such a space, we may compensate for some dynamical effects of diffusion that were missed by the classical Zappalà (1990) approach.

This work is divided as follows: in the second section we see how the main belt appears in frequency space. In the third section we discuss the HCM method originally proposed by Zappalà (1990), introduce a metrics to apply the HCM in frequency space, and discuss the role that asteroid colors play in identifying asteroid families via the "extended metrics" of Bus \& Binzel (2002), and its extension to the new HCM in frequency space. In the fourth section we apply our method to four asteroid families, namely Eos, Koronis, Eunomia, and Vesta, and compare the results to those obtained with the classical methods. Finally, we present our conclusions in the fifth section.

\section{The main belt seen in frequency space}

Secular resonances are commensurabilities that involve the frequencies of the asteroid longitude of perihelion $g$, node $s$ and the fundamental frequencies of planetary theories $g_{i}=\dot{\varpi}_{i}$ and

${ }^{3}$ We used synthetic proper elements and frequencies obtained numerically and publically available at the AstDyS site http://hamilton.dm.unipi.it/cgi-bin/astdys/astibo. The reason for using the numerically obtained proper elements rather than analytical ones will be explained in Sect. 3.2.
Table 1. The values of the planetary frequencies (in "/yr) used in this article.

\begin{tabular}{cc}
\hline \hline Name & Freq. ["/yr] \\
\hline$g_{5}$ & 4.257 \\
$g_{6}$ & 28.243 \\
$g_{7}$ & 3.093 \\
$g_{8}$ & 0.673 \\
& \\
$s_{6}$ & -26.345 \\
$s_{7}$ & -2.996 \\
$s_{8}$ & -0.693 \\
\hline
\end{tabular}

$s_{i}=\dot{\Omega}_{i}$, where $i$ is a suffix that indicates the planets ( 5 for Jupiter, 6 , for Saturn, etc.). The frequencies associated with such resonances have to satisfy the relationship

$p \cdot g+q \cdot s+\sum_{i}\left(p_{i} \cdot g_{i}+q_{i} \cdot s_{i}\right)=0$

where the integers $p, q, p_{i}, q_{i}$ have to fulfill the D'Alembert rules of permissible arguments: the sum of the coefficients must be zero and the sum of coefficients of nodal longitudes frequencies must be even. The critical combinations (1) that involve only the frequency of the asteroid perihelion are often referred to as "pericenter resonances" and those with only frequency of the asteroid node as "node resonances".

The relevance of the sequence of resonances $z_{k}=k(\mathrm{~g}-$ $\left.g_{6}\right)+\left(s-s_{6}\right)(k=1,2,3$, etc. $)$ was first investigated by Milani \& Knežević $(1992,1994)$ and later confirmed by Vokrouhlický et al. (2002, 2006a,b), Carruba et al. (2007b) for the $z_{1}$ resonance, and by Carruba et al. $(2005,2007 \mathrm{~b})$ for the $z_{2}$ resonance. In contrast to mean-motion resonances, secular resonances usually have complicated three-dimensional structures in the proper $a-e-\sin (i)$ space and are not easily represented in a plane. Figure 1 displays the locations of the $z_{k}$ resonances, of the pericenter resonance $g-2 g_{6}+g_{5}$, and of the node resonance $s-s_{6}-g_{5}+g_{6}$ computed for proper $e=0.10$ (the two curves refer to resonances computed for values of $e$ to within \pm 0.025 of the central values) with the program frek.f of Milani \& Knežević $(1994)^{4}$ using the values of planetary frequencies listed in Table 1. The location and shape of the secular resonances change significantly for different values of the proper $e$, making it hard to visualize how asteroid families members may interact with such resonances.

The reason for this behavior is that the secular resonances are not separable in the $(a, e, i)$ space; that is, the position of a resonance depends on all three elements. To overcome this problem, we project asteroids in different space, that of the proper frequencies $(n, g, s)$, where the mean-motion and secular resonances are separable. According to nonlinear perturbation theories, the proper frequencies are functions of the proper elements and the transformation from proper elements to proper frequencies is one-to-one if the Kolgomorov's non-degeneracy condition is satisfied (see Ferraz-Mello 2007). In this space, meanmotion resonances mostly depend on the proper frequency $n$, while secular resonances depend only on the proper $(g, s)$ frequencies and appear as lines in such a plane. Figure 2 displays

4 The analytic approach used by Milani \& Knežević (1994) may fail in some cases to predict the accurate location of some of the nonlinear secular resonances, especially near powerful mean-motion resonances, where the values of proper $g$ are significantly affected. While determining the exact location of those resonances is a task better dealt by numerical methods (Carruba et al. 2007b), we believe that this approach is justified for illustrative purposes. 

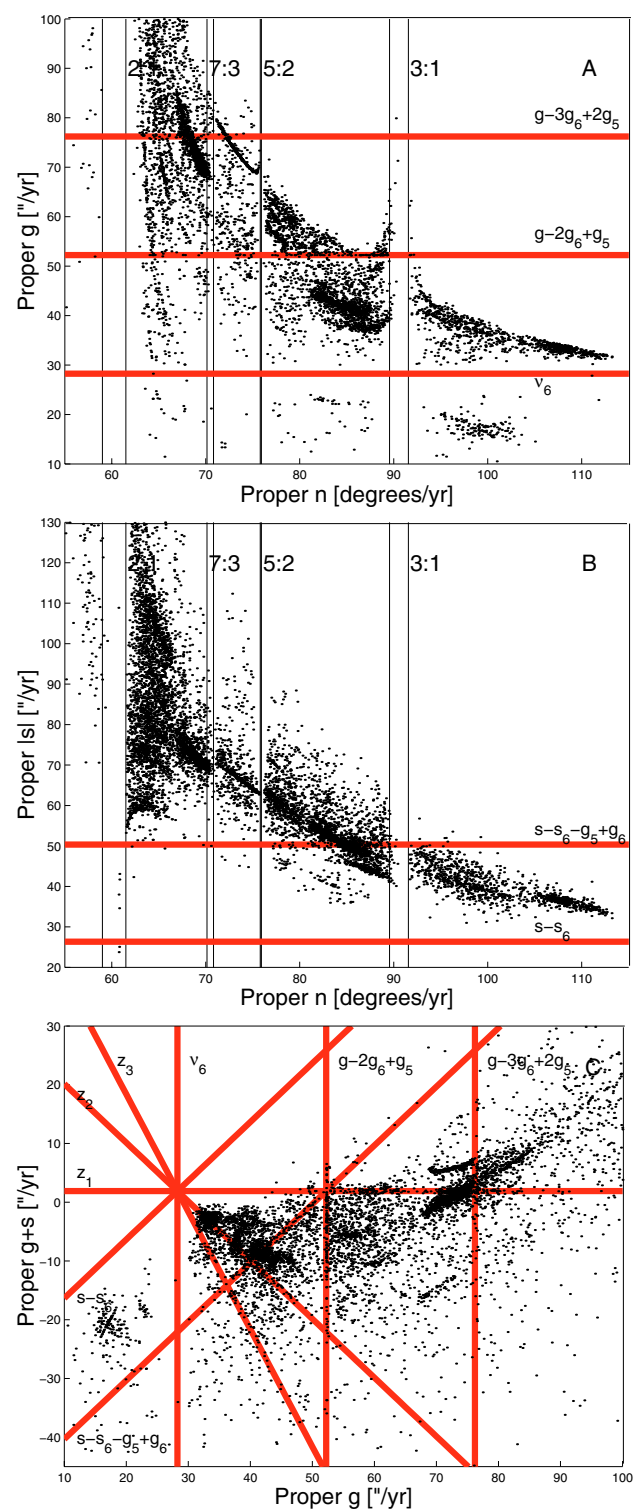

Fig. 2. Projection in the proper $(n, g)(\operatorname{panel} \mathbf{A})),(n,|s|)($ panel B) $)$, and $(g, g+s)($ panel $\mathbf{C}))$ planes of all main belt asteroids with absolute magnitude $\mathrm{H}$ lower than 13. In panels $\mathbf{A}$ ) and $\mathbf{B}$ ), vertical lines display the locations of mean-motion resonances, while horizontal lines show the positions of secular resonances of pericenter and node, respectively. See the text for a discussion of the meaning of the lines in panel C).

the location in the $(n, g),(n,|s|)$, and $(g, g+s)$ planes of the asteroids with available numerical elements and absolute magnitudes lower than 13 .

We start by analyzing the projection in the $(n, g)$ plane. According to the third Kepler law, the proper motions are proportional to the semi-major axes to the power of $-3 / 2$. Therefore, higher values of $n$ correspond to lower values of $a$. Generally, the evolution of proper $g$ as a function of $n$ tends to follow a line of negative slope, but the values of $g$ are strongly affected by powerful mean-motion resonances such as the $3: 1$, the $5: 2$, and the 2:1 resonances with Jupiter (vertical lines in Fig. 2, panel A). As can be seen in panel A of Fig. 2 the values of proper $g$ tend to increase near powerful mean-motion resonances, especially near the $3: 1$ and $2: 1$. This is explained by the appearance of small divisors of the form $\left(k n-l_{j}\right.$ ) (where $k$ and $l$ are integers) in the expression of proper $g$ computed with the analytical perturbation theory of Milani \& Knežević (1994). The effect is also present for the case of the $s$ frequency (see also Knežević et al. 1991), although it is less evident. The behavior of $g$ near powerful mean-motion resonances introduces some difficulties in the asteroid families determination via frequency analysis and will be discussed in more detail in Sect. 3.2 and in Appendix B.

The $(n, g)$ plane is the ideal plane for plotting the location of mean-motion and pericenter resonances. In panel A of Fig. 2 the vertical lines display the main two-body mean-motion resonances with Jupiter and the horizontal lines display the location of four pericenter resonances. The $g-g_{6}$ resonance (known as $v_{6}$ ) is one of the most powerful resonances in the main belt, and it is very efficient in removing any captured asteroid in a short time. The $g-2 g_{6}+g_{5}$ and the $g-3 g_{6}+2 g_{5}$ are two resonances of pericenter. Milani \& Knežević (1994) have shown that these resonances have the strongest effect on the stability of asteroid proper elements. The reader may want to compare the simple shape of the $g-2 g_{6}+g_{5}$ resonance in the $(n, g)$ plane, computed for all possible values of the asteroid eccentricity, with the complicated shape of the resonance in the $a-\sin (i)$ plane (Fig. 1), computed for a small range proper $e$ values. From the comparison, the advantages of using frequency planes are obvious.

We should also point out that secular resonances involving the $g_{5}$ frequency usually present a multiplet structure with the $g_{7}$ fundamental frequency. This is due to the fact that the difference between $g_{5}$ and $g_{7}$ is less than 1.1 "/yr, which Milani \& Knežević (1994) set as the limit for resolving two near secular resonances in proper element space. The values of these two frequencies are so close because the perihelia of Jupiter and Uranus are in fact anti-aligned: $\varpi_{5}-\varpi_{7}$ oscillates around $180^{\circ}$ with a period of 1.1 Myr (Milani \& Nobili 1984). This explains why several nonlinear secular resonances that we observe in the main belt are overlapping. For simplicity, in this work we will only list the resonance involving the jovian frequency when referring to a secular resonance involving the $g_{5}$ frequency, such as the $g-2 g_{6}+g_{5}$. We assume that the reader is aware that nearby resonances involving Uranus frequency $g_{7}$, such as the $g-2 g_{6}+g_{7}$, $g-2 g_{6}+2 g_{5}-g_{7}$, etc., are also present in the region.

Figure 2B shows a projection of the main belt asteroids in the $(n-|s|)$ plane. The proper $s$ frequency is always negative and has a slope as a function of $n$ that is almost opposite to that of $g$. Thus, following the approach of Knežević et al. (1991) we plot the absolute value of the proper frequency $s$ for graphical purposes. As in Fig. 2A, we show the location of the main meanmotion resonances (vertical lines) and of the most powerful secular resonances of node (horizontal lines). In contrast to the case of proper $g$, the values of proper $s$ are not strongly affected by nearby mean-motion resonances, but roughly follow an inclined line until reaching the $2: 1$ resonance.

Finally, Fig. 2C shows a projection of the main belts asteroids in the $(g, g+s)$ plane. Because the slope of $g$ with respect to $n$ is almost opposite that of $s$ with respect to $n$, this representation allows displaying main belt population as parallel to the $x$-axis. In this figure, vertical lines display the locations of perihelion resonances, horizontal lines show resonances that contain the $g+s$ combination, and inclined lines display the location of node resonances and resonances involving other linear combinations of $g$ and $s$. An interesting thing that can be observed in Fig. $2 \mathrm{C}$ is that all the $z_{k}$, the $v_{6}$, and the $s-s_{6}$ resonances pass through the point with coordinates $g=28.243$ " $/ \mathrm{yr}$ and $g+s=1.898 " / \mathrm{yr}$. This is caused because at this point $g=g_{6}$ and $g+s=g_{6}+s_{6}$, and the resonant arguments of all these resonances are simultaneously equal to zero. The location in the proper elements space of this point corresponds to a strongly 


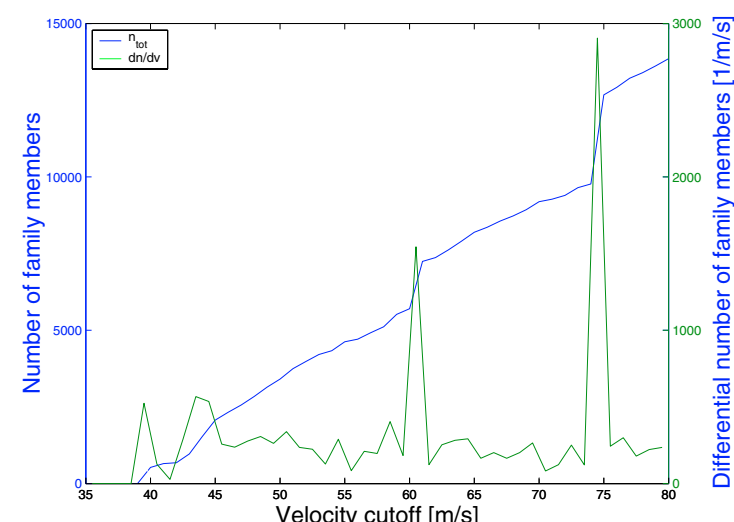

Fig. 3. The number of Eunomia family members (in blue) and the differential number of family members (in green) as a function of the velocity cutoff used in Eq. (2). For $v_{\text {cutoff }}=40 \mathrm{~m} / \mathrm{s}$, the first family members are found, while for $v_{\text {cutoff }}=61 \mathrm{~m} / \mathrm{s}$ the family coalesces with other nearby families such as the Adeona family. For $v_{\text {cutoff }}=74 \mathrm{~m} / \mathrm{s}$, the family coalesces with the local background. In this work we assume that $v_{\text {cutoff }}=55 \mathrm{~m} / \mathrm{s}$ can be a reasonable value for determining the family.

unstable domain inside the $v_{6}$ resonance, at the intersection with the $s-s_{6}$ resonance, and is free of real objects populations.

\section{The hierarchical clustering method in frequency and color spaces}

In this section we present various methods, published and original, of determining asteroid families. We start by revising the classical HCM of Zappalà (1990).

\subsection{The classical hierarchical clustering method}

As can be seen in Fig. 1, asteroid proper elements are clustered in some cases in small regions of space. Following the Zappalà (1990) approach, asteroid families are identified with the following procedure: given an individual asteroid the distance between this object and the other one is computed. If the distance is less than a threshold limit $\left(d_{\text {cutoff }}\right)$, the new object is added to the list. The procedure is repeated until no new family member is found.

A critical point in this procedure is related to the choice of a reasonable metric in the three-dimensional element space. In Zappalà et al. (1990), the distance is defined as

$d=n a \sqrt{k_{1}(\Delta a / a)^{2}+k_{2}(\Delta e)^{2}+k_{3}(\Delta \sin (i))^{2}}$,

where $n$ is the asteroid mean motion; $\Delta x$ the difference in proper $a, e$, and $\sin (i)$; and $k_{1}, k_{2}, k_{3}$ are weighting factors, defined as $k_{1}=5 / 4, k_{2}=2, k_{3}=2$ in Zappalà et al. (1990). Other choices of weighting factors are possible and yield similar results.

The reason for the choice of the value of the weighting factors resides in how Zappalà et al. (1990) modeled the distance in proper element space (see Appendix A). Their idea was essentially to preserve what at the time was believed to be the pristine ejection velocity field of asteroid families. When dealing with distances in the frequency space, we believe a similar approach to the one used by Zappalà et al. (1990) can no longer be used: first, because we now know that the current orbits of members of asteroid families do not reflect the original ejection velocity field, but have probably been modified by several effects (Yarkovsky and YORP effect, diffusion in mean-motion and secular resonances, and the repeated effect of close encounters with massive asteroids, etc.), especially for old families. And second contrary to what was believed in 1990 , we now know that the asteroid semi-major axis is one of the quantities that has undergone the largest variation since the families' formations. Another approach must be found, and will be discussed in the following sections.

Another problem with the classical HCM is the choice of the velocity cutoff. There is no a priori value that can be set, and the velocity cutoff is usually a function of the number of asteroids present in the local background, which also depends on the number of asteroids available in the constantly updated database of asteroid proper elements of Milani \& Knežević (1994). The typical procedure is to find the family for several values of the velocity cutoff, until the family coalesces with the local background, enclosing members of other known asteroid families; then a value that is $90 \%$ of this critical value is used, so as not to include too many asteroids not necessarily linked with the family.

To illustrate this procedure, we applied this method to the Eunomia family and our results are shown in Fig. 3, where we found that $v_{\text {cutoff }}=55 \mathrm{~m} / \mathrm{s}$ is a reasonable choice for the cutoff. For differential number of family members, we mean the number of family members that are added to the family when the cutoff is increased by a fixed quantity (step); for the case of Fig. 3 we used a step of $1 \mathrm{~m} / \mathrm{s}$. A metric that includes the proper frequencies would have to work with different values of the cutoff, and a procedure similar to the one discussed here for the Eunomia family should be applied to this extended metric as well.

\subsection{The hierarchical clustering method in the frequency space}

As discussed in the previous section, the space of proper frequencies $n, g, s$ is the most adequate for identifying secular resonances. A natural question that therefore arises is why not to search for asteroid families in such a space. For this purpose, we need to find an appropriate metric in the frequency space, evaluating the typical distances in frequencies between neighbors in the region of known asteroid families. We applied the classical HCM to various asteroid members of large asteroid families, such as Vesta, Eunomia, Koronis, etc, and computed the differences in proper elements and proper frequencies for their neighbors. Figure 4 shows the histograms of such differences when the classical HCM is applied to (15) Eunomia with a cutoff of $160 \mathrm{~m} / \mathrm{s}$. If we measure $n$ in degree/yr and $g$ and $s$ in "/yr, typical differences for neighbors in families all have the same order of magnitude of 0.05 degrees or " per year.

Since the differences in proper $n, g$, and $g+s$ all have the same order of magnitude, we may define the metric as

$d_{2}=\sqrt{h_{1}\left(\frac{\Delta n}{h_{0}}\right)^{2}+h_{2}(\Delta g)^{2}+h_{3}(\Delta(g+s))^{2}}$

where $h_{0}$ is a normalization factor of dimension 1 degree/", and the simplest choice for the $h_{i}(i=1,3)$ weights is to take them all equal to 1 . The distance in frequency space then has the units of "/yr. We tested other values of the $h_{i}$ coefficients (in the range 0.5-5.0), obtaining similar results.

A problem that might arise by using Eq. (3) is that it might identify artificial clusters near secular resonances. These artificial clumps are formed because the $\simeq 2-10$ Myr averaging procedure used by Milani \& Knežević (2003), to numerically determine the $g, n$, and $s$ frequencies bring all objects into the center 


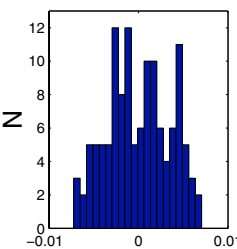

$(\Delta \mathrm{a}) / \mathrm{a}$
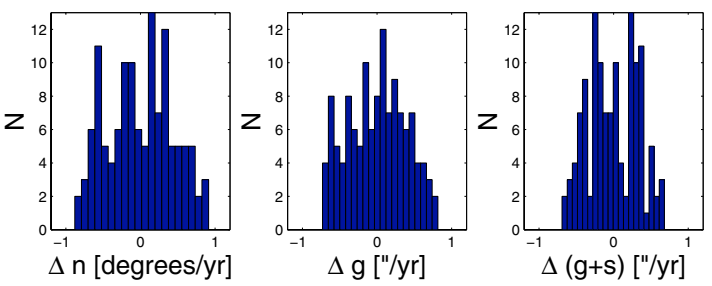

Fig. 4. The changes in proper elements $(\Delta a) / a, \Delta e, \Delta \sin (i)$ (top panels) and proper frequencies $\Delta n, \Delta g, \Delta s$ (bottom panels) for neighbors of (15) Eunomia computed with the classical HCM and a cutoff of $160 \mathrm{~m} / \mathrm{s}$. Results are similar for other asteroids in different regions of the asteroid belt. Note how the changes in proper frequencies all have the same order of magnitude of 0.05 degrees or " per year.

(zero-degrees of libration) of the resonance and produce artificially dense clusters (see Fig. 4 of Milani \& Knežević 2003, and discussion therein). An example of this effect might be seen on the right side of Fig. 1, where a little clump of data points near the $g-2 g_{6}+g_{5}$ resonance is observed. Any new clump or family identified with the frequency approach should therefore be tested by using the classical HCM or other methods, and an analysis of the dynamics in the region should be performed, before the new family can be accepted as such.

Another limitation of the metric given in Eq. (3) is that it fails to recognize as family members objects very close to powerful mean-motion resonances, where the behavior of $g$ as a function of $n$ diverges from a linear one. In the neighborhood of such resonances, asteroids that are close in the proper element space may be very distant in frequency space. A way to partly circumvent this difficulty is to introduce a metric of proper elements and frequencies. This metric, which will be discussed in the Appendix B, may connect some of the critical asteroids that a pure frequency metric may fail to connect. In some cases, however, the change in $g$ is so steep that even this five-dimensional metric fails to connect asteroids across powerful mean-motion resonances. Also, the five-dimensional metric may fail to recognize family members that have drifted far away from the family in proper element space, while we shall see in Sect. 4 that the frequency metric is a powerful tool for detecting asteroids that were captured in nonlinear secular resonances. For simplicity, we decided to solely use the frequency metric and to discuss its limitations on a case to case basis. We believe that losing some peripheral objects is an acceptable price to pay in order to gain the powerful insights on the dynamical evolution of families that the frequency metric allows. But we acknowledged this is a problem yet to be solved.

Finally, Bus \& Binzel (2002) introduced a method to take asteroid colors into account in the family determination. We will discuss this method and its extension to a frequency space in the next subsection.

\subsection{The role of colors}

The Sloan Digital Sky Survey Moving Object Catalog, hereafter SDSS MOC3 lists astrometric and photometric data for asteroids observed by the 2.5-m Sloan telescope located at Apache Point Observatory, in Sunspot, New Mexico. To date (3rd release), the survey has determined the positions, brightness, and fivecolor CCD photometry of 204305 moving objects (Ivezić et al., 2001). The 67636 unique moving objects detected by the survey (i.e., about $28 \%$ of the total) have been matched (Jurić et al. 2002) to known asteroids listed in the ASTORB file (Bowell et al. 1994). The flux reflected by the detected objects was measured almost simultaneously in five bands (measurements in two successive bands were separated in time by $72 \mathrm{~s}$ ) with effective wavelengths 3557 angstrom ( $u$ band), 4825 angstrom ( $g$ band), 6261 angstrom ( $r$ band), 7672 angstrom ( $i$ band), and 9097 angstrom ( $z$ band), and with $0.1-0.3-\mu \mathrm{m}$ band widths (Fukugita et al. 1996).

This data has been used recently by Nesvorný et al. (2004) to classify asteroids according to their taxonomic types and to determine asteroids families in the space of proper elements and colors simultaneously. Bus \& Binzel (2002) introduced the following extended metrics in this space:

$d_{3}=\sqrt{d^{2}+C_{P C}\left[\left(\delta P C_{1}\right)^{2}+\left(\delta P C_{2}\right)^{2}\right]}$.

Where $d$ is given by Eq. (2), $P C_{1}$ and $P C_{2}$ are the two first principal components (we determined them using the approach described in Roig \& Gil-Hutton (2006), $\delta P C_{i}=\left|P C_{i}(1)-P C_{i}(2)\right|$ with $i=1,2$, and $C_{P C}$ is a numerical factor, usually set equal to $10^{6}$, if $v$ is measured in $\mathrm{m} / \mathrm{s}$ (values in a range between $10^{4}$ and $10^{8}$ were tested by the authors without significantly changing the robustness of the results).

We applied this extended metric to the several asteroid families, and the results will be discussed in the next section. As in Nesvorný et al. (2004), we found that, while the extended HCM method in itself does not provide robust concentrations in the extended proper element and color space that could help in identifying new families, the method is excellent to identify family "halos", i.e., populations of peripheral family members that were not joined with the rest of the family with the standard HCM method. In Carruba et al. (2007b) we applied this method to the Eunomia family and found a halo of objects connecting this family with two objects suspected and confirmed to be of the $\mathrm{V}$ taxonomic type. This was very helpful for determining the possible origin of these two asteroids.

Following Bus \& Binzel (2002) we also define a metrics of colors and frequency in the form:

$d_{4}=\sqrt{\left(d_{2}\right)^{2}+D_{P C}\left[\left(\delta P C_{1}\right)^{2}+\left(\delta P C_{2}\right)^{2}\right]}$,

where $D_{P C}$ is a numerical factor, that we empirically set equal to 200 , so as to give comparable results for the typical differences in proper frequencies and the typical differences in principal components. Other values in the range 10-1000 were also tested, without significantly changing the robustness of the results. A discussion of the application of this method to various asteroid families will be given in the next section.

\section{An application to selected asteroid families}

In the next subsections we apply our methods to four asteroid families: Eos, Koronis, Eunomia, and Vesta. While we used our new method to re-derive the major families in the main belt, we prefer to only show families known in the literature to have strong interactions with nonlinear secular resonances, so as to emphasize the advantages of using the new method. In general, we concentrate on the comparison of families obtained with 

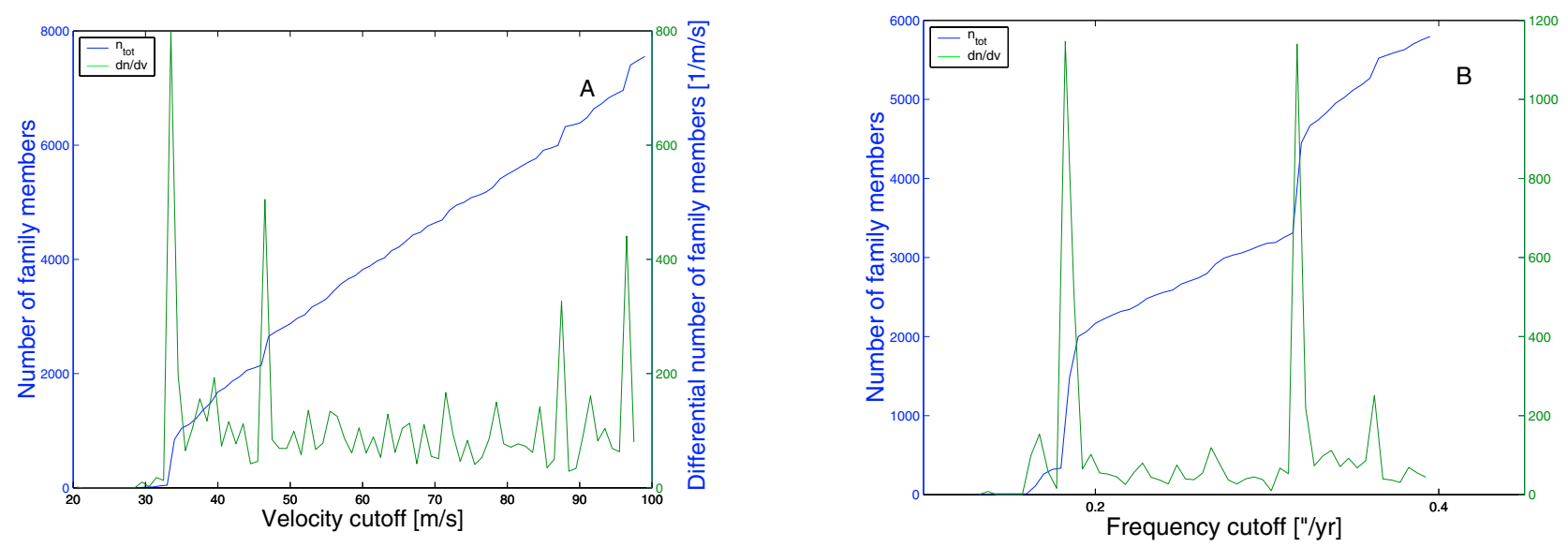

Fig. 5. The number of Eos family members and the differential number as a function of the velocity (left panel) and frequency (right panel) cutoff.

the classical HCM (Eq. (2)) and the frequency metric (Eq. (3)). Results obtained by using the color metrics (Eqs. (4), (5) will be discussed briefly at the end of the four sub-sections.

We will start by analyzing families in the outer main belt and proceed in the inward direction.

\subsection{The outer belt}

\subsection{1. (221) Eos}

The Eos asteroid family is the third most populous, after Themis and Koronis, and its dynamics have been the subject of a thorough recent study by Vokrouhlický et al. (2006). Most of its members belong to the characteristic $\mathrm{K}$ spectral type. The family is bounded by the $7 / 3$ mean-motion resonance with Jupiter. It is crossed by the $9 / 4$ mean-motion resonance, and strongly interacts with the $z_{1}$ nonlinear secular resonance. We start by determining the family with the classical and frequency method and by revising the distribution of mean-motion resonance in the region.

At a velocity cutoff of $34 \mathrm{~m} / \mathrm{s}$, the classical family starts to agglomerate neighbors of (221) Eos (Fig. 5), at $47 \mathrm{~m} / \mathrm{s}$ the family connects to asteroids beyond the $9 / 4$ resonance with Jupiter, and at $87 \mathrm{~m} / \mathrm{s}$ the family coalesces with the near Veritas family. To be consistent with the choice made by Vokrouhlický et al. (2006), we choose a velocity cutoff of $62 \mathrm{~m} / \mathrm{s}$. The first neighbors of (221) Eos in frequency space are found at a frequency cutoff of 0.165 "/yr. At a frequency of $0.320 " / \mathrm{yr}$, the family connects to asteroids beyond the $9 / 4$ resonance. Finally, for a cutoff of $0.365^{\prime \prime} / \mathrm{yr}$, the family merges with the nearby Veritas family. We choose a cutoff of 0.36 " $/ \mathrm{yr}$ to define the family in frequency space. Figure 6A displays the locations in the proper $g, g+s$ plane of the family member obtained with the two methods. The tail of asteroids obtained with the classical method at very high values of $g$ is formed by asteroids that are close to the 2:1 mean-motion resonance. Due to the strong perturbations in proper $g$ caused by this resonance, the frequency method fails to recognize these bodies as family members (see Appendix B).

The next step is to identify the location in the proper $a-\sin (i)$ plane of the main two-body and three-body resonances. We report the location of the $7 / 3,9 / 4,13 / 6$ two-body resonances with Jupiter and of the $3 J-2 S-1 A$ in Fig. 6 . We then identify all nonlinear secular resonances involving the frequencies of the planets from Jupiter to Uranus up to order 6 and display their location in the proper $(g, g+s)$ plane (see Fig. 6A, where the order of a secular resonance is defined as the sum of the absolute values of the $p, q, p_{i}, q_{i}$ coefficients in Eq. (1)). We find that three secular resonances have the strongest impact on the long-term stability of asteroid proper elements: the $z_{1}$ resonance $\left(\right.$ or $\left.\left(s-s_{6}\right)+\left(g-g_{6}\right)\right)$, the $g-\left(-2 g_{5}+3 g_{6}\right)$ resonance, and the $\left(s-s_{6}\right)-2\left(g_{5}-g_{6}\right)$ resonances. Apart from harmonics of these resonances involving combinations of the $g_{5}$ and $g_{7}$ frequencies, other weaker resonances (such as the $s-3 s_{6}+2 s_{7},\left(s-2 s_{6}+s_{7}\right)-\left(g_{5}-g_{6}\right)$, and the $\left(s-s_{7}\right)+\left(g-g_{7}\right)$ and their harmonics $)$ are also present but generally play a minor role. These results agree with Vokrouhlický et al. (2006).

To accurately draw the center of the nonlinear secular resonances in the $a-\sin (i)$ and $(a-e)$ planes, we adopted the following procedures. For each resonance we identify all asteroids in the range around the resonance frequency $\left( \pm 0.3^{\prime \prime} / \mathrm{yr}\right.$ from the central value) that have values of proper $\sin (i)(a-e$ projection) or $e(a-\sin (i)$ projection) compatible with those of the family, or, in some cases, with those of the peculiar feature (for example a tail, like for the case of asteroids in the $\left.\left(s-s_{6}\right)-2\left(g_{5}-g_{6}\right)\right)$ we were studying in the particular case. We then best-fit a polynomial curve to those objects and identify the center of the resonances with this curve. We discuss this procedure in more detail for each of the resonance we are going to study.

Figure 6B shows an $a-\sin (i)$ projection of the Eos classical and frequency families (for brevity, we do not show an $a-e$ and $e-\sin (i)$ projection of the Eos family, since the $a-\sin (i)$ plane is the most interesting one). As in Vokhrouhlický et al. (2006), we find that the $z_{1}$ resonance (computed for $e=0.08$ ) plays a major role in shaping the Eos family. The tail of objects at $a \simeq 3.05$ and $\sin (i) \simeq 0.2$ identified by the classical methods as members that drifted via the Yarkovsky force in the $z_{1}$ are also found with the new approach. The new metric, however, allows us to identify a stream of objects leaving the Eos family toward lower values of $\sin (i)$ via the $g-2 g_{5}+3 g_{6}$ and the $\left(s-s_{6}\right)-$ $2\left(g_{5}-g_{6}\right)$ resonances that the classical method failed to recognize as family members. Other streams of objects leaving the family are associated to harmonics of these two resonances involving the $g_{7}$ frequency, such as $g+3 g_{6}-2 g_{7}$, the $\left(s-s_{6}\right)-2\left(g_{7}-\right.$ $g_{6}$ ) or other possible combinations of $g_{5}$ and $g_{7}$. We believe the identification of these secular resonances as possible pathways for dynamical diffusion, obtained without performing extensive numerical simulations of the asteroid orbital evolution, is one of the most important result of our method.

Another interesting result found with the frequency metrics regards four asteroids, (20845) 2000 UY102, (21211) 1994 PP36, (33780) 1999 RU171, and (62948) 2000 VE32, currently 

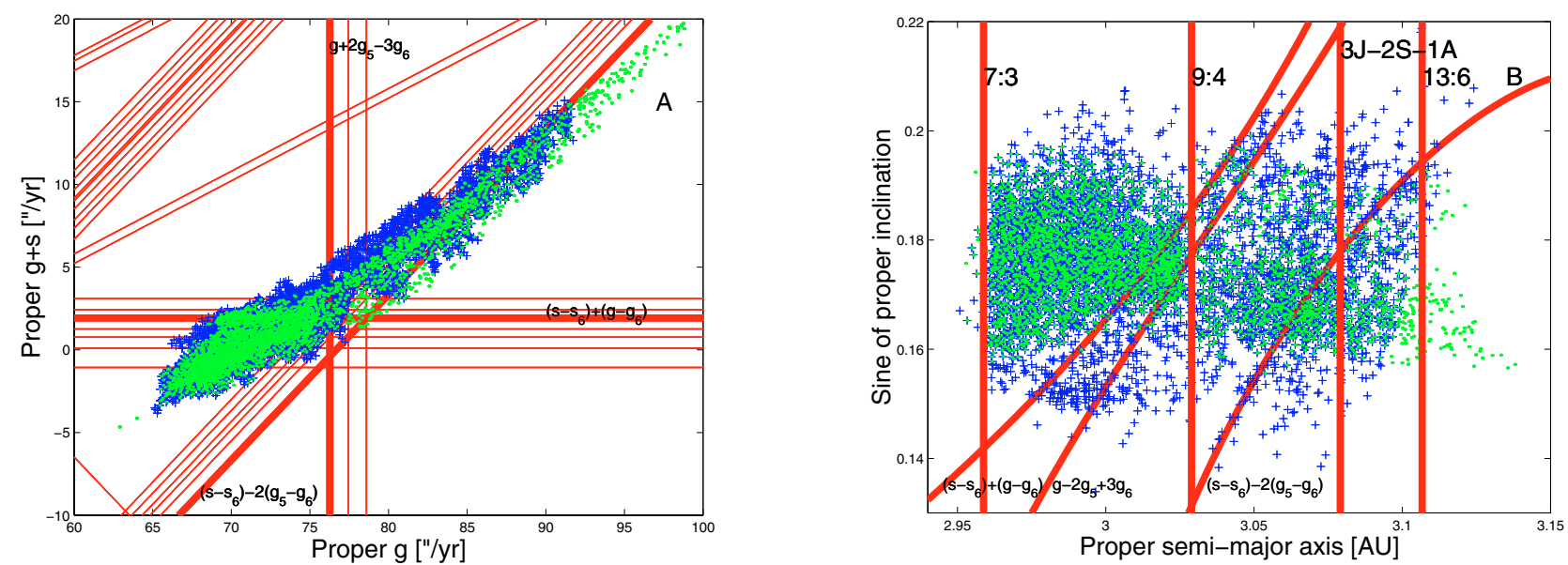

Fig. 6. A $(g, g+s)$ and $(a, \sin (i))$ projection of the Eos family obtained with the classical HCM (green dots), and with the frequency HCM (blue crosses). Secular resonances shown in $(a, \sin (i))$ planes are identified by thicker lines in the $(g, g+s)$ plane (we have identified all secular resonances of order up to 6).
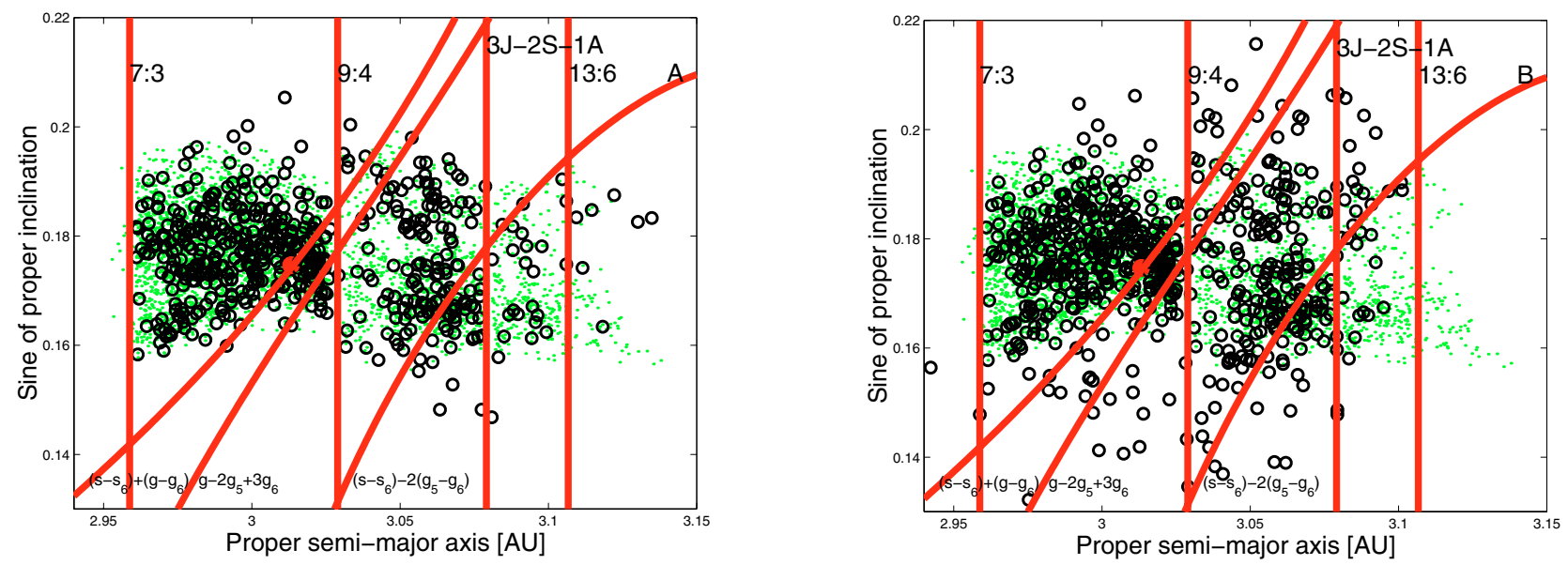

Fig. 7. An $a-\sin (i)$ projection of the members of the Eos family found with the classical approach (green dots), with the metric of colors and proper elements (black circles, left panel) and with the metric of color and frequencies, (black circles, right panel). The full large red dot displays the location of (320) Katharina. The other symbols have the same meaning as those in Fig. 6B.

inside the $z_{1}$ resonance and at low values of proper $e$ and $\sin (i)$. Vokrouhlický et al. (2006) hypothesized that those objects were former members of the Eos family that diffused to their current position due to the interplay of the Yarkovsky effect and the $z_{1}$ resonance. To investigate this hypothesis, they were observed by some of the Vokhrouhlický et al. (2006) authors so as to confirm their taxonomic compatibility with Eos family members ${ }^{5}$. We find that these four asteroids are identified as Eos members by the frequency method, thereby confirming the usefulness of performing an analysis of families in the frequency space.

We also identified the Eos family in the spaces of proper elements and colors (Eq. (4)) and of proper frequencies and colors (Eq. (5)). As the first member for the family, we chose the lowest numbered object in the dynamical family that was also present in the SDSS catalog: (320) Katharina (Carruba et al. 2007b). We repeated the analysis of the dependence of the number of families members on the cutoffs and we chose to work with a cutoff of $189 \mathrm{~m} / \mathrm{s}$ (metric of colors and proper elements) and of $1.78^{\prime \prime} / \mathrm{yr}$ (metric of colors and frequencies), respectively.

5 The first three objects have a $\mathrm{T}$ spectral type and are compatible with Eos family membership, while the fourth was most likely an interloper.
While the family in the color and proper element space essentially follows the same orbital distribution of the family obtained with the classical approach, a very interesting result regarding the metric of colors and frequencies is that it recognizes objects that drifted in the $z_{1}, g-2 g_{5}+3 g_{6}$ and $\left(s-s_{6}\right)-2\left(g_{5}-g_{6}\right)$ resonances toward lower values of proper $\sin (i)$ as family members.

Also, while the metric of color and frequencies identifies all features already identified by the frequency metric, the advantage of the frequency metric is that it is not limited to the asteroids currently present in the SDSS catalog, thereby identifying a larger number of family members than the metrics of color. It is evident in Fig. 7 that many objects identified by the frequency metric as family members were not in the SDSS catalog, and did not appear in the "color" families. We believe this argument clearly shows the advantage of frequency metrics: while some of the evolutionary features of asteroid families could be identified using methods already in the literature, such as the metric of Bus \& Binzel (2002), only families obtained in frequency space can give a more complete picture of the family dynamical evolution. We clarify this point further with the analysis of the other three families we focused on. 

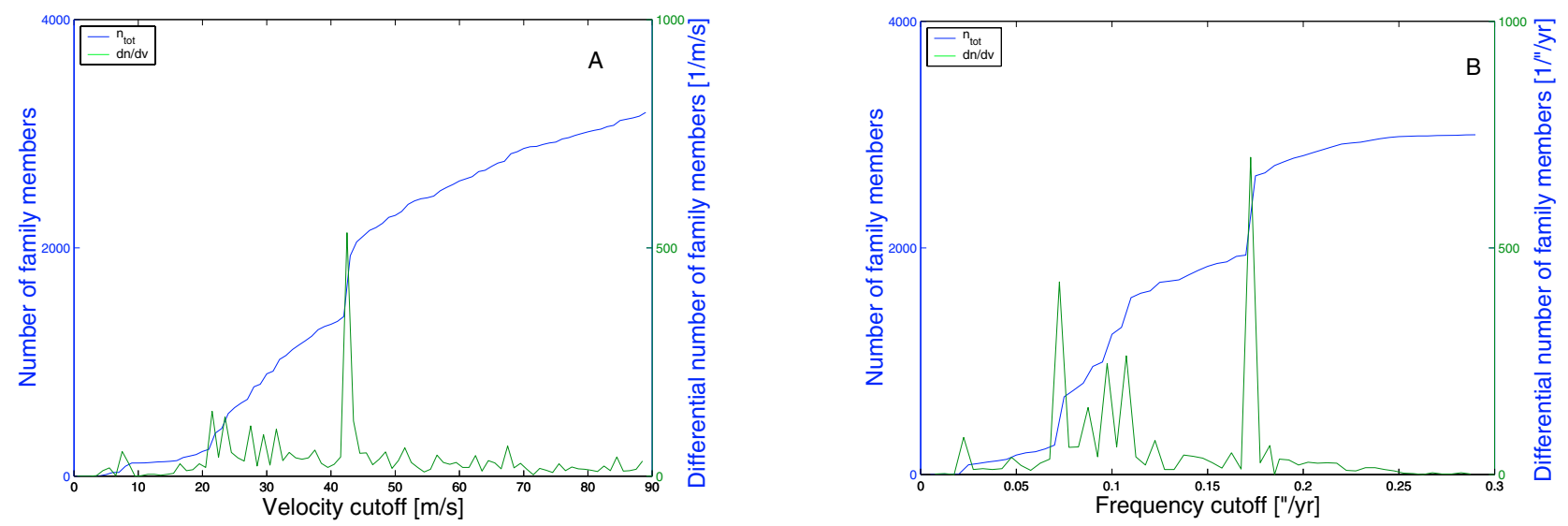

Fig. 8. The number of Koronis family members and the differential number as a function of the velocity (left panel) and frequency (right panel) cutoff.
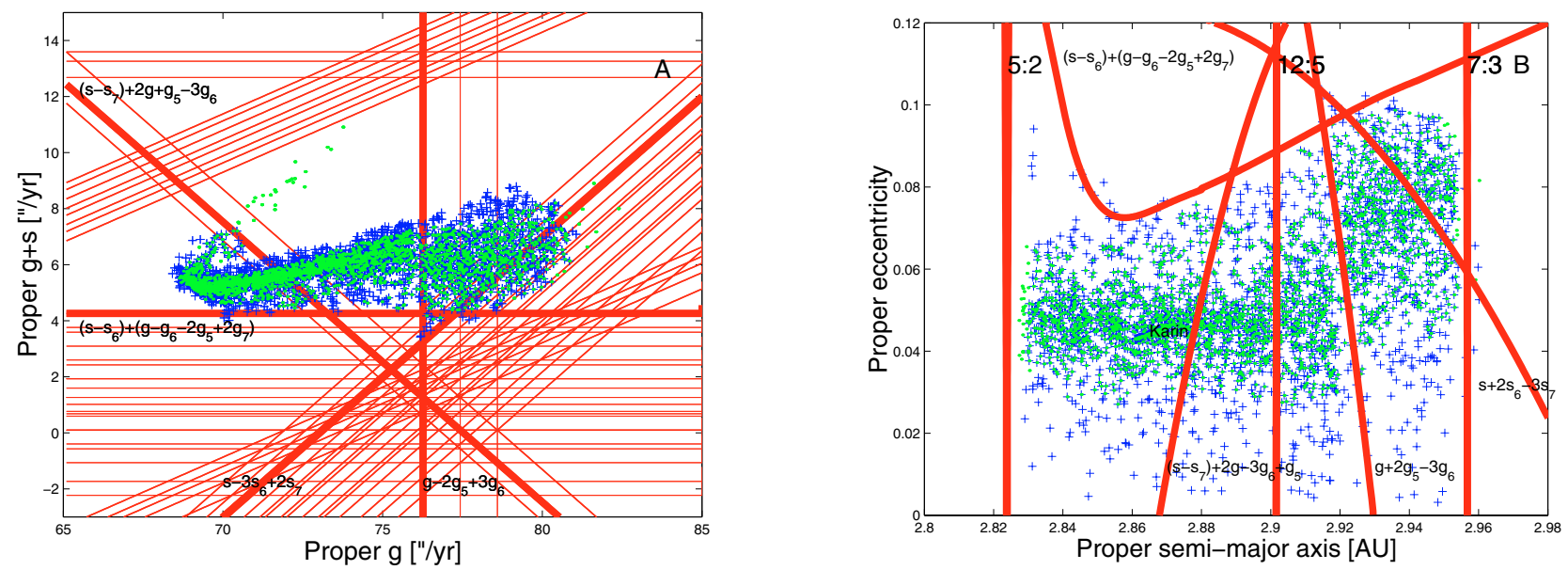

Fig. 9. A $(g, g+s)$ and $(a-e)$ projections of the Koronis family obtained with the classical HCM (green dots) and with the frequency HCM (blue crosses). The Karin cluster is shown at $a \simeq 2.865 \mathrm{AU}$.

\subsection{2. (158) Koronis}

As for the Eos family, we start by identifying the Koronis family with the classical and the frequency metrics. Figure 8 displays the number of Koronis family members and the differential number as a function of the velocity (left panel) and frequency (right panel) cutoff. For $v_{\text {cutoff }}=43 \mathrm{~m} / \mathrm{s}$, the family found with the classical method connects to the asteroids beyond the $g+2 g_{5}-3 g_{6}$ secular resonance, as already found by Bottke et al. (2001), while the family connects to asteroids beyond the $7: 3$ mean-motion resonance with Jupiter for $v_{\text {cutoff }}>60 \mathrm{~m} / \mathrm{s}$. To be consistent with the family used by Bottke et al. (2001), we choose a cutoff of $56 \mathrm{~m} / \mathrm{s}$. The right panel of Fig. 8 displays the number of Koronis family members as a function of the frequency cutoff. For $v_{\text {cutoff }}=0.075^{\prime \prime} / \mathrm{yr}$, the first nucleus of the family is found. Around $v_{\text {cutoff }}=0.100 " / \mathrm{yr}$, a series of secondary features inside the Koronis families start to be identified, including the Karin cluster of Nesvorný and Bottke (2004). For $v_{\text {cutoff }}=0.175^{\prime \prime} / \mathrm{yr}$, the family connects with asteroids beyond the $g+2 g_{5}-3 g_{6}$ secular resonance, and the family is essentially saturated for values of $v_{\text {cutoff }}>0.250 " / \mathrm{yr}$, i.e., increasing the cutoff to values of $v_{\text {cutoff }}=0.300 " / \mathrm{yr}$ only causes the family to grow by six new members. For this reason, we choose to work with the family defined by $v_{\text {cutoff }}=0.250 " / y$. The classical family so determined is in agreement with that found by Bottke et al. (2001).
The Koronis family is bounded by the 5:2 and 7:3 meanmotion resonances with Jupiter, is crossed by the $12: 5$ resonance, and has a strong interaction with the $g-2 g_{5}+3 g_{6}$ secular resonance (Bottke et al. 2001). The most striking feature of the Koronis family is visible in a $a-e$ projection: asteroids beyond the $g-2 g_{5}+3 g_{6}$ secular resonance have their eccentricity significantly enhanced by the passage through the resonance, thus giving the family a characteristic bi-modal shape (see Fig. 9; for brevity we do not show an $a-\sin (i)$ projection of this family).

We identified all nonlinear secular resonances up to order 8 . We needed to go to a higher order to clarify what caused some of the observed features in the proper element distribution of the frequency family. Figure 9A displays the usual $(g, g+s)$ projection. Apart from the $g-2 g_{5}+3 g_{6}$ secular resonance (and the usual harmonics involving combinations of $g_{5}$ and $g_{7}$ ), we identified three other resonances responsible for diffusing members of the family: the $s-3 s_{6}+2 s_{7}$, the $\left(s-s_{7}\right)+2 g+g_{5}-3 g_{6}$ and the $\left(s-s_{6}\right)+\left(g-g_{6}+2 g_{5}-2 g_{7}\right)$ resonances. The last one is a harmonic of the powerful $z_{1}$ secular resonance. Figure 9B displays an $a-e$ projection of the family, with the location of these resonances computed for the inclination of (158) Koronis. Asteroids that diffused to lower values of proper $e$ did so mostly by interacting with the $g-2 g_{5}+3 g_{6}$ and $\left(s-s_{7}\right)+2 g+g_{5}-3 g_{6}$ secular resonances or their harmonics. The upper side of the frequency family appears to be nicely bounded by the $\left(s-s_{6}\right)+\left(g-g_{6}+2 g_{5}-2 g_{7}\right)$ secular resonances. It was already known that asteroid families 

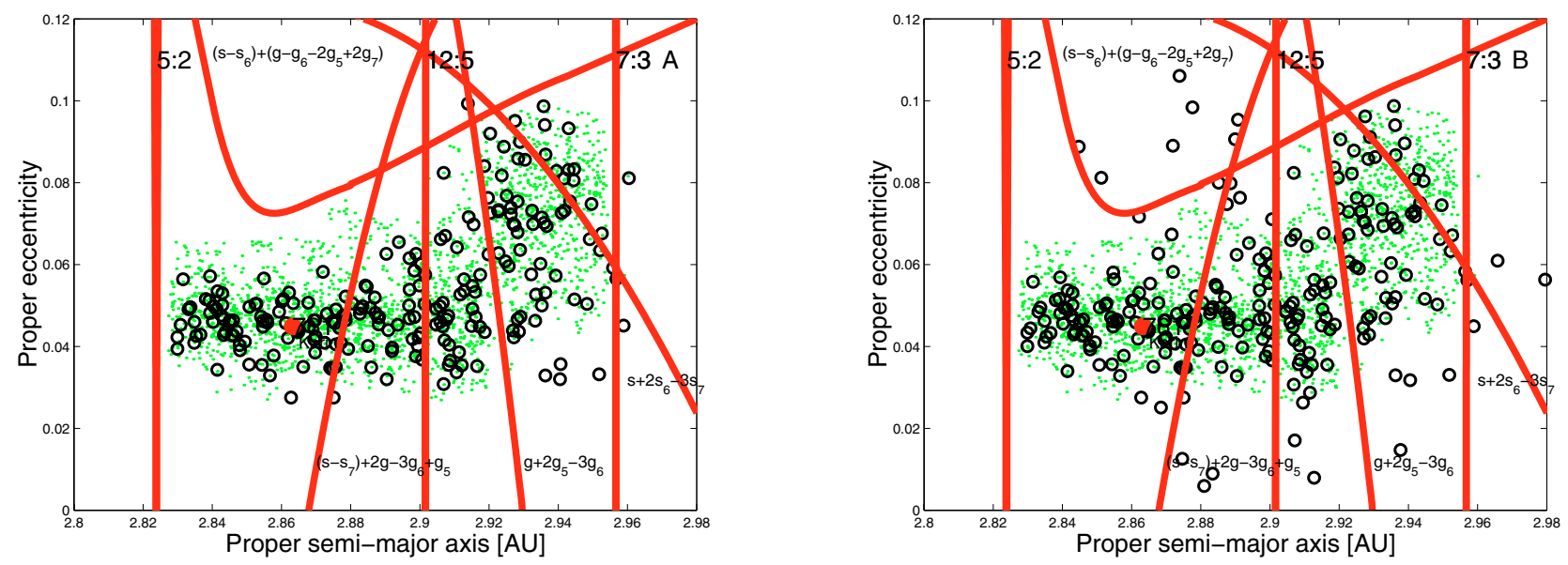

Fig. 10. An $a-\sin (i)$ projection of the members of the Koronis family found with the classical approach (green dots), with the metric of colors and proper elements (black circles, left panel), and with the metric of color and frequencies (black circles, right panel). The full large red dot displays the location of (761) Brendelia. The other symbols have the same meaning as those in Fig. 6B.

were bounded by mean-motion resonances; our results for the Koronis and for the Eunomia families suggest that asteroid families are also bounded by nonlinear secular resonances.

We also performed an analysis of the Koronis family with the color metrics. The metric of color and elements (Fig. 10, left panel) did not reveal new information. We chose the lowest numbered asteroid in the dynamical family that is also present in the SDSS catalog, (761) Brendelia, and determined the family for various values of the velocity cutoff. For a cutoff of $198 \mathrm{~m} / \mathrm{s}$, the family coalesced with the local background, and for lower cutoffs family members essentially followed the same $(a-e)$ distribution of the members of the Zappalà classical family.

Regarding the metric of color and frequencies, results confirm those of the metric of frequency. The family merged with the local background for a frequency cutoff of 1.82 "/yr, so we chose to work with a cutoff of $1.78^{\prime \prime} / \mathrm{yr}$. Apart from a few objects at high eccentricity above the $\left(s-s_{6}\right)+\left(g-g_{6}+2 g_{5}-2 g_{7}\right)$ resonance not found with the frequency metric, all other objects were already present in the list found with Eq. (3).

\subsection{The middle Belt}

\subsection{1. (15) Eunomia}

The Eunomia family is one of the largest families in the middle belt. It is bounded on the right side by the 8:3 mean-motion resonance, but its limit on the left side is not so easily defined when the classical HCM is used. We applied both the classical and the frequency HCMs to this family. Figure 3 shows how the number of member varies as a function of the velocity cutoff, while Fig. 11 show the same as a function of the frequency cutoff. Finally, Fig. 12 displays $(g, g+s)$ and $(a-\sin (i))$ projection of the Eunomia family obtained with both methods.

As can be seen in Fig. 12, the frequency HCM manages to recognize asteroids that drifted in nonlinear secular resonances and that the classical HCM does not recognize as family members. In particular, the left side of the Eunomia family is now strongly bounded by the $\left(g-g_{7}\right)+2\left(s-s_{6}\right)\left(\right.$ and $\left.\left.g-g_{5}\right)+2\left(s-s_{6}\right)\right)$ resonances. Farther on the left, for lower values of $a$, the influence of the near 3:1 mean-motion resonance with Jupiter causes a sudden increase in the values of proper $g$ (see Fig. 2), which also causes an overlap of several secular resonances such as the $z_{1}$, the $g-\left(2 g_{5}-g_{6}\right)$, etc. to occur. We believe that this might be

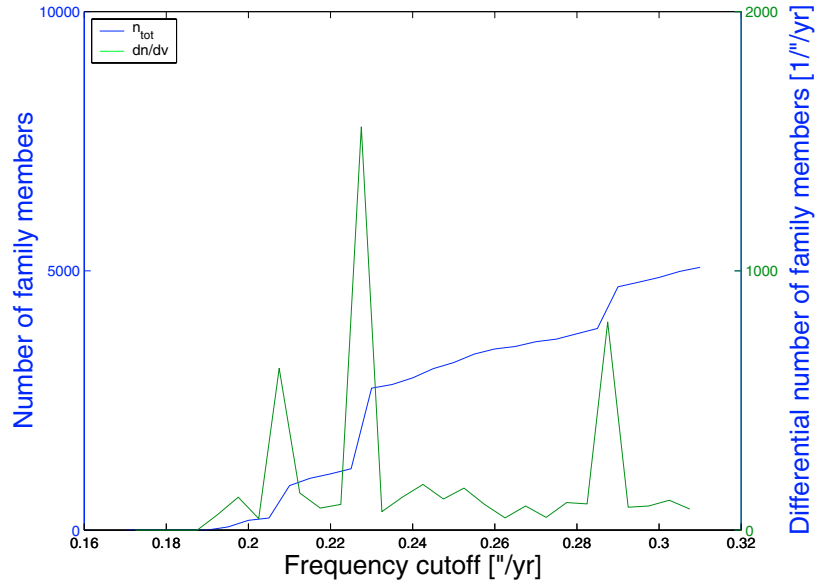

Fig. 11. The number of Eunomia family members (in blue) and the differential number of family members (in red) as a function of the frequency cutoff used in Eq. (3). For $f_{\text {cutoff }}=0.21 " / \mathrm{yr}$, the first family members are found, while for $f_{\text {cutoff }}=0.310 " / \mathrm{yr}$ the family coalesces into the local background. The secondary peak at $f_{\text {cutoff }}=$ 0.285 "/yr is associated with the coalescence of secondary features inside the Eunomia family. In this work we use a frequency cutoff of $0.275^{\prime \prime} / \mathrm{yr}$.

one of the causes for the large-scale instabilities near the boundaries of the 3:1 resonance, which was first studied by Guillens et al. (2002).

The frequency HCM also allowed us to detect a tail of asteroids that drifted in the $\left(s-s_{6}\right)-\left(g_{5}-g_{6}\right)$ resonance. Recently, Carruba et al. (2007b) has hypothesized that (21238) 1995 WV7 and (40521) 1999 RL95, two newly identified V-type objects (Hammergren et al. 2006; Roig et al. 2007) might be explained in the framework of migration from the near Eunomia family via Yarkovsky diffusion in secular resonances. While most of the members of the Eunomia family are S-type asteroids, it has been observed (Nathues et al. 2005) that (15) Eunomia is the remnant of a differentiated or partially differentiated body whose originally pyroxene-enriched crust layer was lost either in the collision that originated the Eunomia family or in a preceding collision (Carruba et al. 2007). We believe the presence of this tail of newly found Eunomia members in the $\left(s-s_{6}\right)-\left(g_{5}-g_{6}\right)$ 

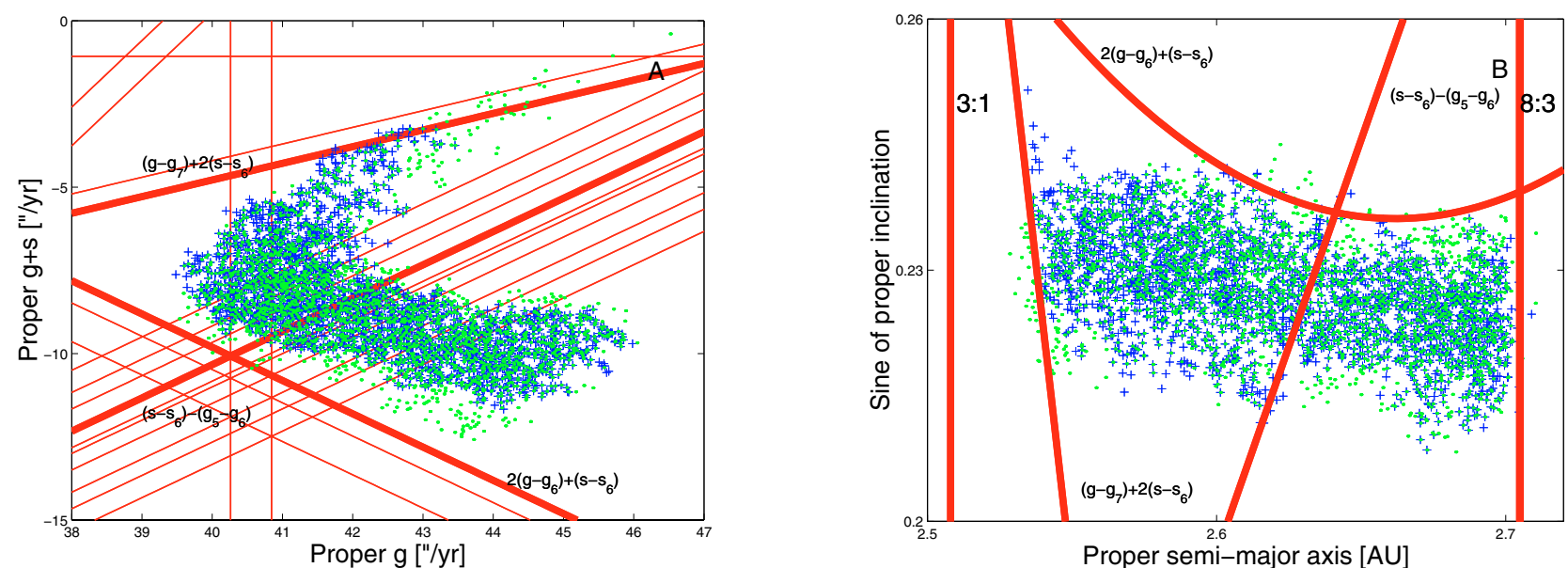

Fig. 12. A $(g, g+s)$, and $(a-i)$ projection of the Eunomia family obtained with the classical HCM (green dots) and of that obtained with the frequency HCM (blue crosses). Note that the objects that diffused in the secular resonances of perihelia and node, such as $\left(g-g_{7}\right)+2\left(s-s_{6}\right)$, and of node, such as $\left(s-s_{6}\right)-\left(g_{5}-g_{6}\right)$, are only identified by the frequency HCM as family members. The family is bounded by the $8: 3$ mean-motion resonance on the right and by the $\left(g-g_{5}\right)+2\left(s-s_{6}\right)$ and $\left(g-g_{7}\right)+2\left(s-s_{6}\right)$ resonances on the left.
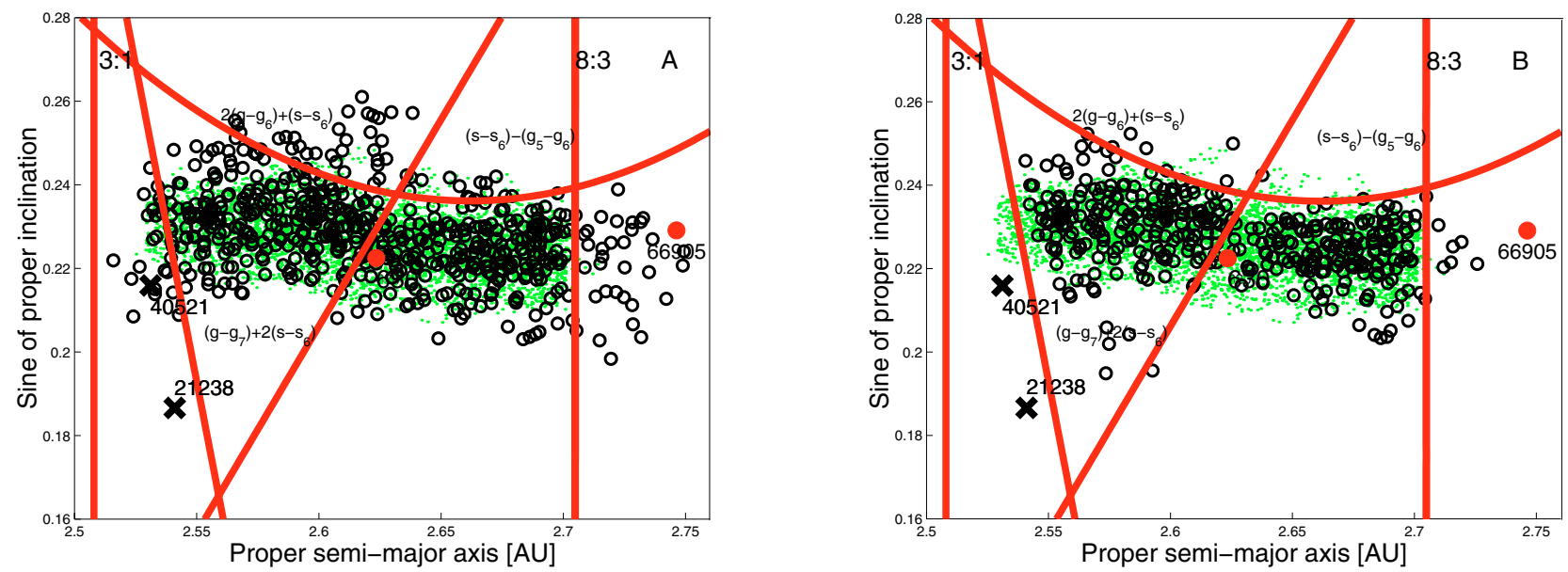

Fig. 13. The $a-i$ projection of (21238) and (40521) (black $\times$ ), and of a V-type candidate identified by Roig \& Gil-Hutton (2006), (66905), large red dot, of members of the Eunomia dynamical family computed with a cutoff of $55 \mathrm{~m} / \mathrm{s}$ (small green dots), and of members of the Eunomia family in the extended space of proper elements and colors, with a cutoff of $229 \mathrm{~m} / \mathrm{s}$ (black circles, left panel). and of members of the family in the extended space of proper frequencies and colors, with a cutoff of 1.1 " $/ \mathrm{yr}$ (black circles, right panel). The full red dot at $a \simeq 2.62$ AU displays the location of (630) Euphemia. The other lines have the same meaning as in Fig. 12B.

resonance further confirms the hypothesis of (21238) 1995 WV7 being one of the last surviving fragments of the longlost crust of the parent body of (15) Eunomia (Carruba et al. 2007).

Finally, the upper side of the Eunomia family seems to be defined by the influence of the strong $z_{2}\left(2\left(g-g_{6}\right)+\left(s-s_{6}\right)\right)$ resonance. The frequency HCM allowed us to detect a tail of asteroids that drifted in such resonance until reaching higher values of $\sin (i)$.

We also performed an analysis of the Eunomia family with the color metrics. We chose (630) Euphemia as the lowest numbered asteroid in the dynamical family also present in the SDSS catalog and determined the family with a velocity cutoff of $231 \mathrm{~m} / \mathrm{s}$ and a frequency cutoff of 1.1 "/yr (beyond that, the family coalesced with the nearby Maria family).

The family found with the metric of color and frequencies confirmed the important role played by the $\left(s-s_{6}\right)-\left(g_{5}-g_{6}\right)$ resonance, as also found in Carruba et al. (2007). The metrics of color also identified a few objects that diffused in the $z_{2}$ resonance and were not found with other methods.

\subsection{The inner belt}

\subsection{1. (4) Vesta}

We start by determining the Vesta family with the classical and with the frequency approaches. Figure 14 shows the number and differential number of family members as a function of the velocity and frequency cutoffs.

The Vesta family in proper elements space coalesces with the background for a cutoff of $74 \mathrm{~m} / \mathrm{s}$ and in frequency space for a frequency of $0.135^{\prime \prime} / \mathrm{yr}$. We therefore choose to use cutoffs of $68 \mathrm{~m} / \mathrm{s}$ and of 0.134 "/yr, respectively. As can be seen in Fig. 15, the frequency method does not connect asteroids to the family beyond values of $g \simeq 41.6^{\prime \prime} / \mathrm{yr}$. This is due to the influence of the near 3:1 mean-motion resonance with Jupiter, which causes a steep increase in the variation of $g$ with respect to $n$. We discuss a possible way to partially solve this problem in the Appendix B.

The next step is to determine the location of the main meanmotion resonances in the region. The Vesta family is bounded by the powerful 3:1 and 7:2 mean-motion resonances with Jupiter and is crossed by the 1:2 resonance with Mars. Among the 

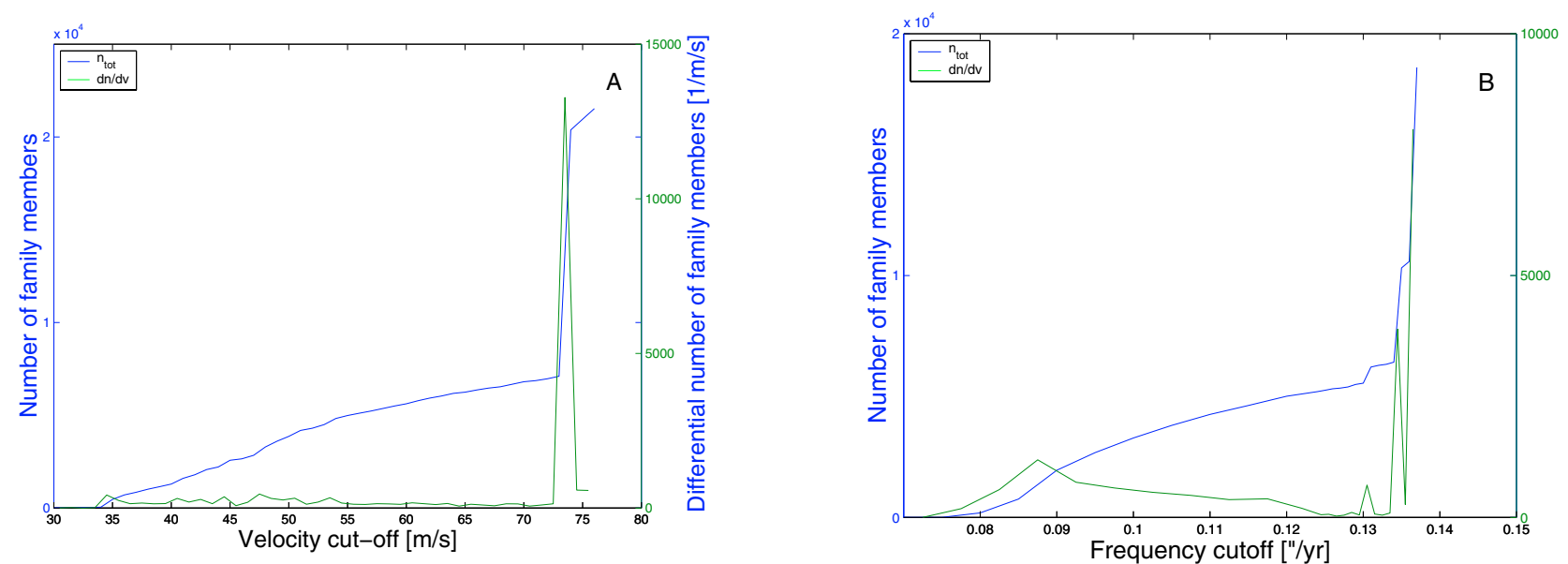

Fig. 14. The number of Vesta family members and the differential number as a function of the velocity (left panel) and frequency (right panel) cutoff.
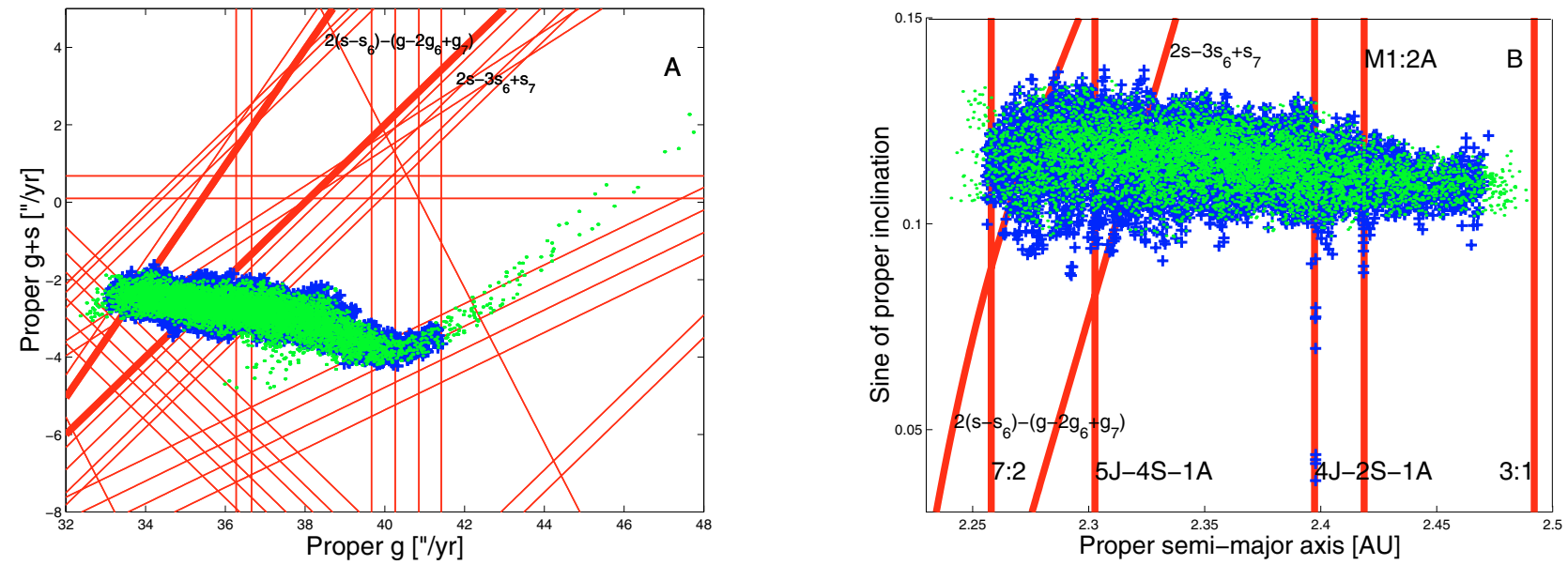

Fig. 15. A $(g, g+s)$ and $(a-\sin (i))$ projections of the Vesta family obtained with the classical HCM (green dots) and obtained with the frequency HCM (blue crosses).

three-body resonances, the $5 \mathrm{~J}-4 \mathrm{~S}-1 \mathrm{~A}$ and the $4 \mathrm{~J}-2 \mathrm{~S}-1 \mathrm{~A}$ are known to play significant dynamical roles (Carruba et al. 2005).

The role of secular resonances in the dynamics of members of the Vesta family is generally minor when compared to that of mean-motion resonances. Apart from two resonances, the $2 s-3 s_{6}+s_{7}$ and the $2\left(s-s_{6}\right)-\left(g-2 g_{6}+g_{7}\right)$ and their harmonics involving the $g_{5}$ and $g_{7}$ frequencies, nonlinear secular resonances do not generally perturb the family members significantly. In this case, however, the frequency approach to family determination is very important for identifying the effect of diffusion via mean-motion resonances. The $a-\sin (i)$ projection of the Vesta classical and frequency families displayed in the right panel of Fig. 15 shows how the frequency approach was able to identify objects that migrated in the $4 J-2 S-1 A$ and the $1 M: 2 A$ mean-motion resonances toward lower values of proper $\sin (i)$ as family members. Since most of the V-type asteroids (asteroids with the same spectral type of most of the Vesta family members for which a spectral classification is available) outside the Vesta family are actually found at lower values of proper $\sin (i)$ than the family, we believe our method provides a possible explanation for the origin of some of these objects, without the need to perform a time-costly simulation with symplectic integrators.

Also, we found that the family obtained in the frequency space includes three of the V-type asteroids outside the classical family (3536) Schleicher, (4977) Rauthgundis, and (7148)
Reinholdbien), and ten objects identified by Roig and Gil-Hutton (2006) as having SDSS albedos compatible with a V-type spectral classification: (6563) Steinheim, (7484) Dogo Onsen, (14323) 1979 MV1, (19809) Nancyowen, (35718) 1999 FE34, (53950) 2000 GX56, (56381) 2000 EN43, (61068) 2000 LR6, (64311) $2001 \mathrm{UQ32}$, and (103401) 2000 AW130. We believe that the fact that our approach to family determination detects fugitives from asteroid families directly is an important validation of our method.

We also performed an analysis of the Vesta family with the color metrics. We pick (1979) Sakharov as the lowest numbered asteroid in the dynamical family also present in the SDSS cata$\log$, and determine the family for various values of the velocity and frequency cutoffs. We chose to work with a velocity cutoff of $217 \mathrm{~m} / \mathrm{s}$ and with a frequency cutoff of $0.86^{\prime \prime} / \mathrm{yr}$. The families coalesced with the local background for cutoffs of $218 \mathrm{~m} / \mathrm{s}$ and 0.87 "/yr, respectively.

Apart from a few isolated objects, mostly beyond the 7:2 resonance and along the $5 \mathrm{~J}-4 \mathrm{~S}-1 \mathrm{~A}$, the metrics of colors did not provide any significant new information on the orbital diffusion of Vesta family members. It is interesting to notice that the families in frequency space coalesced with the background mostly at values of low $\sin (\mathrm{i})(\sin (i)<0.1)$ and low $a<2.3 \mathrm{AU}$, following the two strong secular resonances in the region $\left(2 s-3 s_{6}+s_{7}\right.$ and $\left.2\left(s-s_{6}\right)-\left(g-2 g_{6}+g_{7}\right)\right)$. 

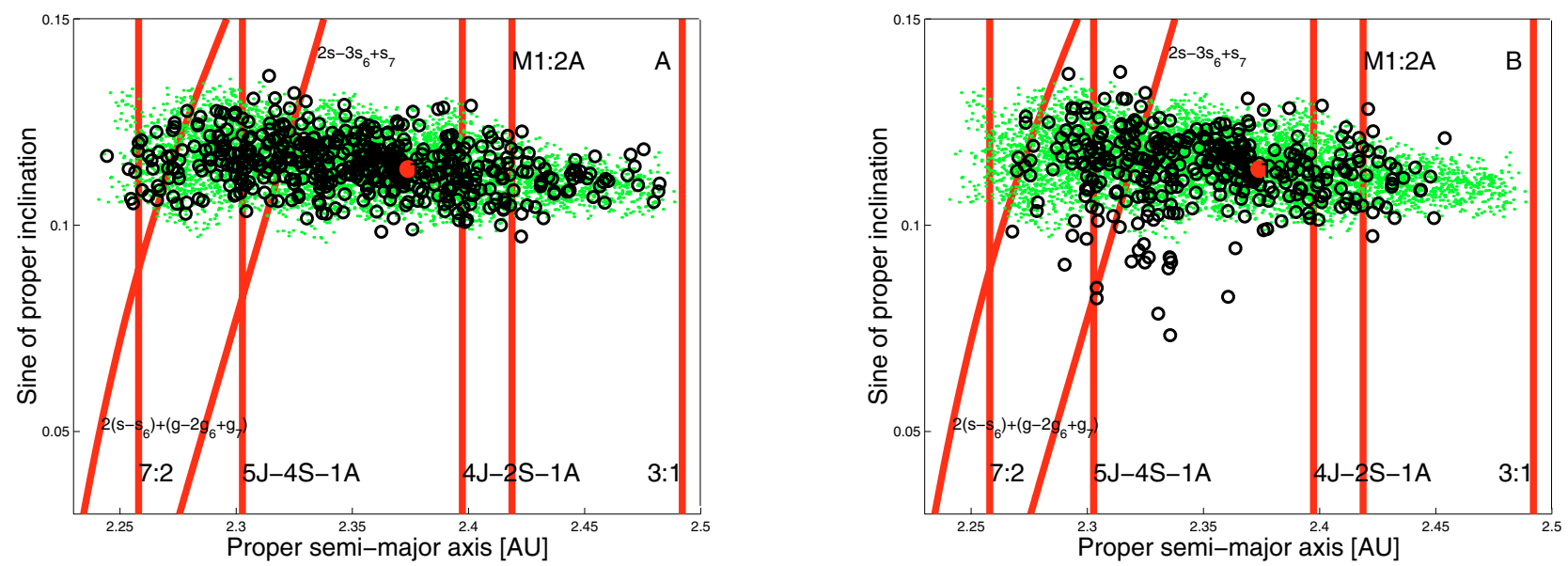

Fig. 16. An $a-\sin (i)$ projection of the members of the Vesta family found with the classical approach (green dots), with the metric of colors and proper elements (black circles, left panel), and with the metric of color and frequencies, (black circles, right panel). The full large red dot displays the location of (1979) Sakharov. The other symbols have the same meaning as in Fig. 6B.

\section{Conclusions}

In this work we obtained asteroid families from a different perspective than the one used traditionally. Since several papers in the last years have shown the importance that secular resonances have played in shaping the orbits of members of asteroid families, we decided to analyze asteroid families in proper frequency space, where the complicated three-dimensional structure of secular resonances may be more easily represented. For this purpose, we did the following:

- We introduced a distance metric in the proper frequency space $(n, g, g+s)$.

- Following the approach of Bus \& Binzel (2002), we extended the metric of asteroid colors and proper elements to the frequency space.

- We applied the new approaches to identifying members of four of the largest asteroid families in the inner, middle, and outer belt namely Vesta, Eunomia, Eos, and Koronis.

One benefit of analyzing the dynamics in the $g, g+s$ plane is that secular resonances are very easily represented as lines in this plane, in contrast to the complicated three-dimensional structure that such resonances have in the $a, e, \sin (i)$ space. This makes this plane ideal for understanding the dynamical effects that secular resonances have produced on asteroid families.

The most striking result we obtained regards what Bus and Binzel (2002) defines as "halo objects", i.e., objects on the boundaries of the families that were not connected with the Zappalà $(1990,1994)$ approach. The hierarchical clustering method in frequency space is a very efficient way to identify family members that migrated because of the interplay between the Yarkovsky effect and nonlinear secular resonances. Our analysis of the four asteroid families has confirmed results obtained previously through numerical simulations with symplectic integrators that included the Yarkovsky effect and, in some case, discovered new possible paths of migration. By identifying "halo objects", we believe that our method may be very useful for identifying possible targets for observational campaigns. Also, simulations with symplectic integrators to account for the diffusion of such objects may provide limits on asteroid families' ages (see also Carruba et al. 2005, 2007a,b).

Another interesting result regards the family boundaries. It was already known that asteroid families were bounded by powerful mean-motion resonances, but our analysis showed they are also bound by secular resonances such as the $z_{2}$ resonance for the Eunomia family and a harmonic of the $z_{1}$ resonance (the $\left(s-s_{6}\right)+\left(g-g_{6}-2 g_{5}+2 g_{7}\right)$ for the Koronis family. Also, the fact that in the region near powerful mean-motion resonances, such as the 2:1 and the 3:1, the values of $g$ suffer a steep increase produces an overlapping of secular resonances in these regions. We believe this may be one of the causes of the instability of orbits observed by Guillens et al. (2002) in the proximity of the $3: 1$ resonance.

The steep behavior of $g$ (and, in a less measure, of $s$ ) in these regions also causes a limitation to our approach. Our metric fails to connect objects near powerful mean-motion resonances. We show that other approaches, such as the metrics of frequencies and proper elements described in the Appendix B, may partially solve this problem, but at the price of losing objects that drifted far in secular or mean-motion resonances. Since, in this work, we were mostly interested in determining possible migration paths away from asteroid families, we only concentrated on the use of the metric of frequencies.

To conclude, while numerical simulations will always be needed to confirm the viability of the evolution of asteroids drifting away from families (Carruba et al. 2007b), we believe that the new approach to asteroid family identification provides a fast way to hypothesize about the diffusion mechanisms of former asteroid families' members. We consider this to be the major new result of this work.

\section{Appendix A: The Zappalà metrics}

The metrics described in Eq. (2) was originally devised by Zappalà et al. (1990) in order to most accurately preserve the alleged pristine ejection velocity field of asteroid families. At the time, it was believed that the current proper orbital element distribution of asteroid families reflected the ejection velocity field formed at the time of the catastrophic disruption of asteroid families.

Following Brouwer (1951) and Zappalà et al. (1984), if we neglect terms proportional to the eccentricity, the following relationship between change in proper elements and velocity at infinity holds:

$2 \Delta V_{N} / n a=\Delta a / a$,

$\Delta V_{R} \sin (f) / n a+2 \Delta V_{N} \cos (f) / n a=\Delta e$, 

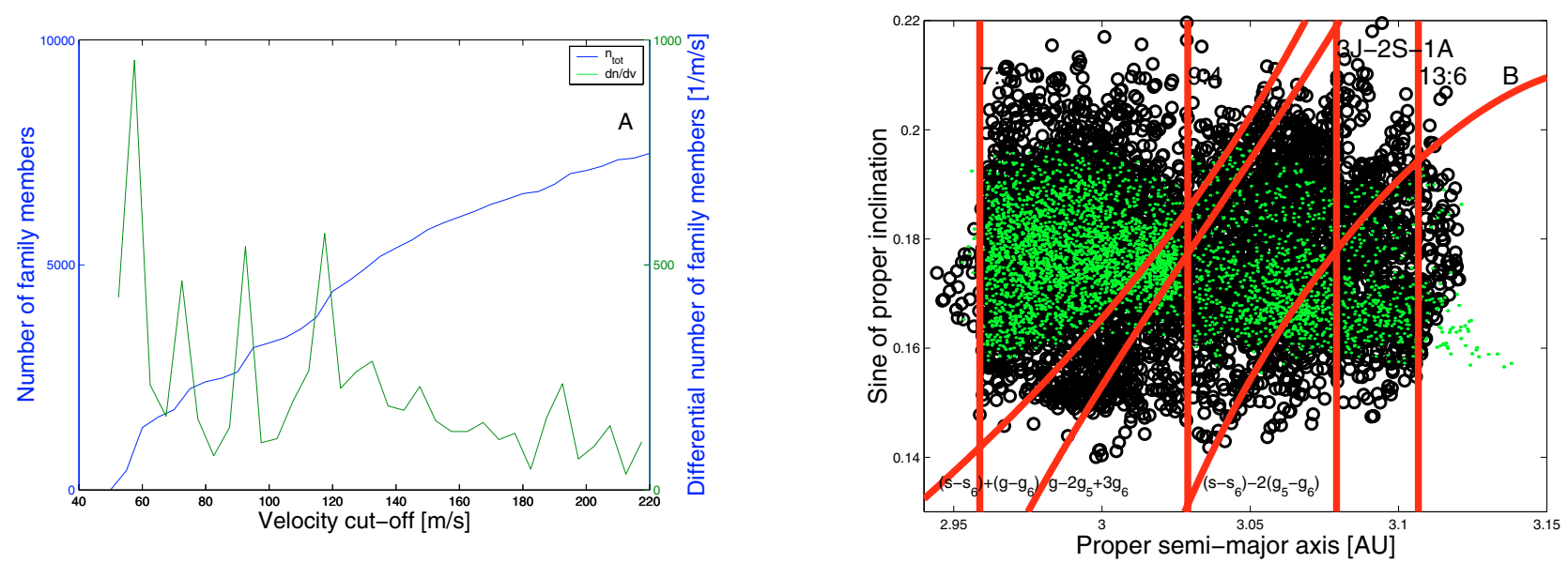

Fig. B.1. Left panel: the number and differential number of the Eos family found with the metric of proper elements and frequencies (Eq. (11)) as a function of the velocity cutoff. In the right panel we show an $a-\sin (i)$ projection of the members of the Eos family found with the classical approach (green dots), and with the metric of proper elements and frequencies (black circles).

$\Delta V_{W} \cos (\omega+f) / n a=\Delta i$,

where $a, e, i, \omega$, and $f$ are the osculating semi-major axis, eccentricity, inclination, argument of perihelion, and true anomaly of the parent body, $V_{N}, V_{R}, V_{W}$ are the components if the velocity at infinity in the along-track, radial, and out-of-plane directions, respectively (the other quantities have the same meaning as in Eq. (2)).

Even with $f$ and $\omega+f$ unknown, if we choose a distance function in the proper element space in the form

$\Delta V=n a \sqrt{k_{1}(\Delta a / a)^{2}+k_{2}(\Delta e)^{2}+k_{3}(\Delta i)^{2}}$,

with coefficients $k_{1}, k_{2}, k_{3}$ of order unity, the metric will give an order of magnitude estimate of the velocity increase causing separation of the two orbits.

By squaring Eq. (6), averaging over $f$ and $f+\omega$, and then substituting in the $\Delta$ elements, Zappalà et al. (1990) obtained

$\Delta v=\sqrt{x\left\langle\Delta V_{N}{ }^{2}\right\rangle+y\left\langle\Delta V_{R}{ }^{2}\right\rangle+z\left\langle\Delta V_{W}{ }^{2}\right\rangle}$,

with

$x=\left(4 k_{1}+2 k_{2}\right), y=k_{2} / 2, z=k_{3} / 2$.

As the authors noticed, there was no choice for the $k_{i}(i=1,2,3)$ coefficient that yielded $x=y=z=1$. The choice of $k_{1}=$ $5 / 4, k_{2}=2, k_{3}=2$ was made to give a greater weight to the $\Delta V_{N}$ component, and therefore to the proper semi-major axes that were known with higher accuracy than proper eccentricities and inclinations.

\section{Appendix B: A metric of proper elements and frequencies}

As discussed in Sect. 3.2, the frequency metric may fail to connect to the bulk of the family asteroid near very powerful mean-motion resonances. Recently, Nesvorný et al. (2006) and Nesvorný and Vokrouhlický (2006) have introduced a five dimensional metric to investigate the database of asteroid osculating orbital elements in order to identify very recent asteroid families. In their work, the authors used a metric of the form:

$$
\begin{aligned}
\left(\frac{d}{n a}\right)^{2}=k_{1}\left(\frac{\Delta a}{a}\right)^{2}+k_{2}(\Delta e)^{2}+k_{3}(\Delta \sin (i))^{2} & +k_{\Omega}(\Delta \Omega)^{2} \\
& +k_{\varpi}(\Delta \varpi)^{2},
\end{aligned}
$$

where $\Delta \Omega, \Delta \varpi$ are the asteroid separations in longitude of the node and pericenter, respectively, and $k_{\Omega}=k_{\varpi}$ are factors ranging from $10^{-4}$ to $10^{-7}$ (the other symbols have the same meaning as in Eq. (2)). The authors used $k_{\Omega}=k_{\varpi}$ because the secular precession rates of $\Omega$ and $\varpi$ are comparable across the main belt (Brouwer \& Clemence 1961).

Following this example, to solve some of the shortcomings of a pure frequencies' metric, we introduced an extended metric of the form:

$$
\left(\frac{d}{n a}\right)^{2}=k_{1}\left(\frac{\Delta a}{a}\right)^{2}+k_{2}(\Delta e)^{2}+k_{3}(\Delta \sin (i))^{2}+k_{g s}\left((\Delta g)^{2}+(\Delta s)^{2}\right),(1
$$

with $k_{g s}$ a factor going from $10^{-3}$ to $10^{-5}$, with the best result given by $k_{g s}=10^{-4}$. We applied this metric to the two families that are close to the $2: 1$ and $3: 1$ mean-motion resonances, and presented the problems related to the very steep increase in $g$ near such resonances, when the frequency metric was applied: Eos and Vesta. Other approaches that we tried, such as metrics with logarithms of the distances in frequencies or metrics with normalized distances in frequencies (of the form $2\left(g_{1}-g_{2}\right) /\left(g_{1}+g_{2}\right)$, for example) did not produced satisfactory results. Generally, they produced families that rapidly coalesced into the local background.

Figure B.1 displays the number and differential number of Eos family members as a function of the velocity cutoff (left panel) and a $a-\sin (i)$ projection of the family. For $v_{\text {cutoff }}=$ $190 \mathrm{~m} / \mathrm{s}$ the Eos family coalesced with near families, so we choose to work with a cutoff of $185 \mathrm{~m} / \mathrm{s}$. The metric of color and frequencies identified almost all asteroids with $a$ beyond the 13:6 mean-motion resonance with Jupiter and was able to catch most of the family members found by the metric of frequency. It did however failed to identify members at very low and very high $\sin (i)$ that drifted in one of the harmonics of the $\left(s-s_{6}\right)-2\left(g_{5}-g_{6}\right)$. While very minor for the Eos family, this is an effect that has been observed in higher proportions in other families, like the Vesta one.

Figure B.2 displays the number and differential number of Vesta family members as a function of the velocity cutoff (left panel) and a $a-\sin (i)$ projection of the family. We choose to work with a cutoff of $85 \mathrm{~m} / \mathrm{s}$, just below the value for which the family coalesced into the background.

As can be seen in the right panel of Fig. B.2, the metric of proper elements and frequencies actually did connected 

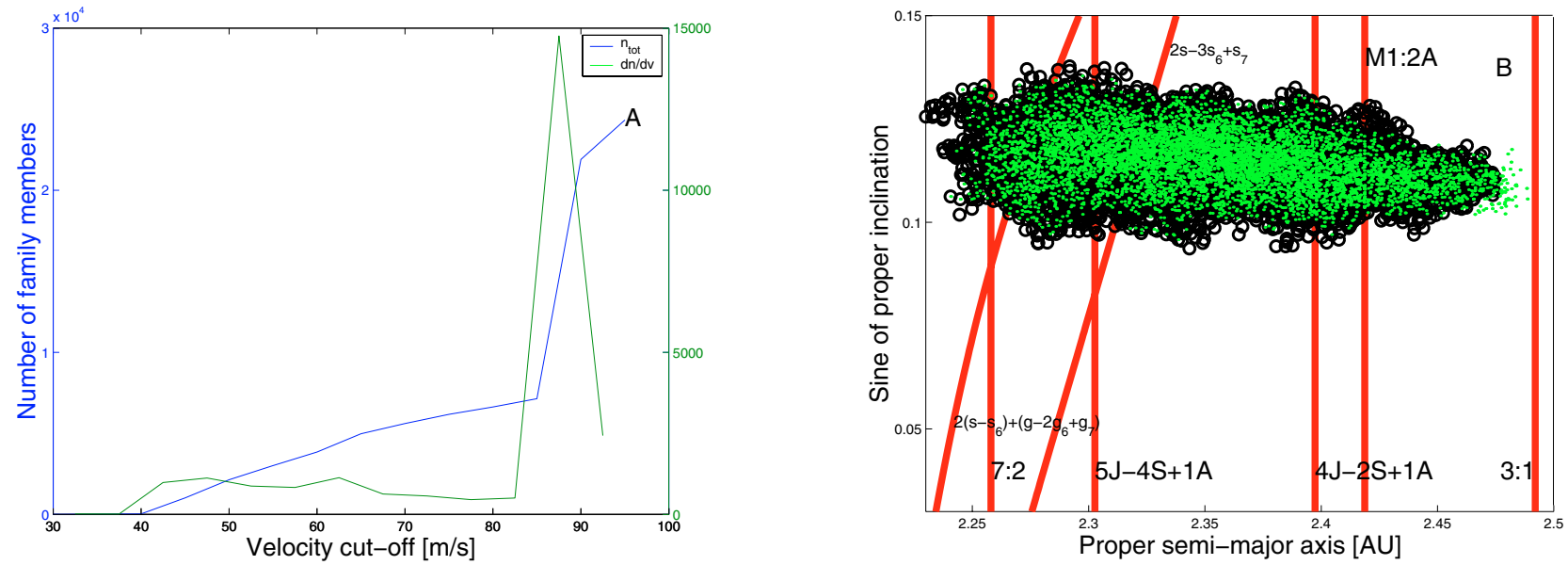

Fig. B.2. Left panel: the number and differential number of the Vesta family found with the metric of proper elements and frequencies (Eq. (11)) as a function of the velocity cutoff. In the right panel we show an $a-\sin (i)$ projection of the members of the Vesta family found with the classical approach (green dots) and with the metric of proper elements and frequencies (black circles).

almost all asteroids up to the neighborhood of the 3:1 resonance. But this was achieved at the cost of losing the asteroids found by the metric of frequencies, which migrated at lower values of proper $\sin (i)$, via the $2 s-3 s_{6}+s_{7}$ secular resonance, possibly the $4 J-2 S-1 A$ three-body resonance, and other resonances (see Fig. 15B). Since in this work we were mostly interested in determining possible migration paths from asteroid families, we decided to mostly use the metric of frequencies and to accept its limitations near powerful mean-motion resonances.

Acknowledgements. We are grateful to David Vokrouhlický for his careful revision of our paper and for various helpful hints and suggestions. The authors would also like to thank the São Paulo State Science Foundation (FAPESP) and the Brazilian Research Council (CNPq), which supported this work via diverse grants and fellowships. The codes used for family determination are available upon request to the first author.

\section{References}

Bendjoya, P., \& Zappalà, V. 2002, Asteroids III (Tucson: Univ. of Arizona Press), 613

Bottke, W. F., Vokrouhlický, D., Brož, M., Nesvorný, D., \& Morbidelli, A. 2001, Science, 294, 1693

Bowell, E., Muinonen, K., \& Wasserman, L. H. 1994, Asteroids III (Tucson: Univ. of Arizona Press), 477

Brouwer, D. 1951, Astron. J. 56, 9

Brouwer, D., \& Clemence, G. M. 1961, Methods of Celestial Mechanics (New York: Academic)

Bus, J. S., \& Binzel, R. P. 2002a, Icarus, 158, 106

Bus, J. S., \& Binzel, R. P. 2002b, Icarus, 158, 146

Carruba, V., Burns, J., Ćuk, A., Bottke, W., \& Nesvorný, D. 2003, Icarus, 162, 308

Carruba, V., Nesvorný, D., Burns, J. A., Ćuk, M., \& Tsiganis, K. 2004, AJ, 128, 1899

Carruba, V., Michtchenko, T., Roig, F., Ferraz-Mello, S., \& Nesvorný, D. 2005, A\&A 441, 819
Carruba, V., Roig, F., Michtchenko, T., Ferraz-Mello, S., \& Nesvorný, D. 2007a, A\&A, 465, 315

Carruba, V., Michtchenko, T., \& Lazzaro, D. 2007b, A\&A, 473, 967

Ferraz-Mello, S. 2007, Canonical Perturbation Theories, Degenerate Systems and Resonances, ed. W. B. Burton, et al. (New York: Springer), 345, 88

Fukugita, M., Ichikawa, T., Gunn, J. E., et al. 1996, AJ, 111, 1748

Gladman, B. J., Migliorini, F., Morbidelli, A., et al. 1997, Science, 277, 197

Guillens, S.A., Vieira Martins, R., \& Gomes, R. S. 2002, AJ, 124, 2322

Hammergren, M., Gyuk, G., \& Puckett, A. 2006 [arXiv:astro-ph/0609420]

Hirayama, K. 1918, Astron. J. 31, 185

Ivezić Z., and 32 colleagues 2001, AJ, 122, 2749

Jurić M., and 15 colleagues 2002, AJ, 124, 1776

Knežević, Z., Milani, A., Farinella, P., Froeschlé Ch., \& Froeschlé, C. 1991, Icarus, 93, 316

Knežević, Z., \& Milani, A. 2000, Celest. Mech. Dyn. Astron., 78, 17

Milani, A., \& Nobili, A. M. 1984, Nature 310, 753

Milani, A., \& Knežević, Z. 1992, Icarus 98, 211

Milani, A., \& Knežević, Z. 1994, Icarus 107, 219

Milani, A., \& Knežević, Z. 2003, A\&A 403, 1165

Nathues A., Mottola, S., Kaasalainen M., \& Neukum, G. 2005, Icarus, 175, 452

Nesvorný, D., \& Bottke, W., 2004, Icarus, 170, 324

Nesvorný, D., \& Vokrouhlický, D. 2006, AJ, 132, 1950

Nesvorný, D., Jedicke, R., Whiteley, R. J., \& Ivezić, Z. 2005, Icarus, 173, 132

Nesvorný, D., Vokrouhlický, D., \& Bottke, W. F. 2006, Science, 312, 1490

O’Brien, D. P., \& Greenberg, R. 2005, Icarus, 178, 179

Roig, F., \& Gil-Hutton, R. 2006, Icarus, 183, 411

Roig, F., Nesvorný, D., Gil-Hutton, R., \& Lazzaro, D. 2007, [arXiv: astro-ph/0707.1012]

Vokrouhlický D. 1999, A\&A, 344, 362

Vokrouhlický D., \& Brož, M. 2002, Modern Celestial Mechanics: from Theory to Applications, 467

Vokrouhlický D., \& Čapek, D. 2002, Icarus, 159, 449

Vokrouhlický D., Brož, M., Morbidelli, A., et al. 2006a, Icarus, 182, 92

Vokrouhlický D., Brož, M., Bottke, W. F., Nesvorný, \& Morbidelli, A. 2006b, Icarus, 183, 349

Zappalà V., Farinella, P., Knežević, Z., \& Paolicchi, P. 1984, Icarus, 59, 261

Zappalà V., Cellino, A., Farinella, P., \& Knežević, Z. 1990, AJ, 100, 2030

Zappalà, V., Bendjoya, Ph., Cellino, A., Farinella, P., \& Froeschlè, C. 1995, Icarus, 116, 291 